九州大学学術情報リポジトリ

Kyushu University Institutional Repository

Estimations after Preliminary Test of Significance and Their Applications to Biometrical Researches

Asano, Choichiro

Shionogi Pharamaceutical Co. | Kyushu University

https://doi.org/10.5109/12991

出版情報: 統計数理研究. 9 (2/3)，pp.1-23，1960-10. Research Association of Statistical Sciences バージョン：

権利関係 : 


\title{
ESTIMATIONS AFTER PRELIMINARY TEST OF SIGNIFICANCE AND THEIR APPLICATIONS TO BIOMETRICAL RESEARCHES
}

\author{
By \\ Chooichiro Asano \\ (Received January 25, 1960)
}

\section{Introduction.}

In many actual cases of statistical inferences used in biometrical and pharmaceutical researches, it may often happen that we have to appeal to a successive process of testing various hypotheses and of estimations of parameters.

In view of our practical uses of statistical inferences, the unwary seems to be left with the impression that some statistical analysis stated in current text-books are, in short, more concerned with inferences by a single use of sample mean or by that of sample variance than with inferences in which sample mean, sample variance and previous informations are used in cooperation with each other. No statistician, who is conformed with the effectiveness of statistical inference, can be allowed to obsure such an obstacle in physical and biological sciences. These situations were pointed out by Kitagawa [1] in the Introduction of his first paper on successive process of statistical inferences.

In most of practical circumstances it seems us to be essentially needed that we should make some inferences on the validity test of observations and some uses of previous informations, before we may appeal to the standard procedures of testing hypothesis or of estimations. General aspects of our statistical procedures in such cases will take some form of pooling data.

It is our standpoint that the formulation of these procedures can be given in a form of successive process of statistical inference, in which (i) our model is " not completely specified" in the sense of Bozivich-BancroftHartley [1] and (ii) the previous information is to be taken into our consideration, under certain conditions discussed in Kitagawa [2].

The object of this paper is to formulate, in the light of successive process of statistical inferences, several important statistical procedures being used in practical situations and to discuss with some merits of these procedures from our view-point. Section 2 is concerned with inferences about the population mean, while Section 3 with those about the population variance. 
In conclusion the author wishes his hearty thanks to Prof. T. Kitagawa for his kind suggestions and encouragement.

\section{$\S 2$. Inferences about population means}

\section{1. Type 1}

Let us consider the situation where we are provided with two samples $O_{n_{1}}$ and $O_{n_{2}}$. The first sample $O_{n_{1}}$ will give us some information which is rather general and stable, while the second sample $O_{n_{2}}$ will yield us another information which is rather special and unstable, but which is more intimately connected with we want to infer. Let us assume that $O_{n_{1}}:\left(x_{11}, x_{12}, \cdots, x_{1 n_{1}}\right)$ gives us an estimate $\bar{x}_{1}$ for the population mean $\mu_{1}$, where $\bar{x}_{1}$ is distributed in $N\left(\mu_{1}, \sigma^{2} / n_{1}\right)$, and let $O_{n_{2}}:\left(x_{21}, x_{22}, \cdots, x_{2 n_{2}}\right)$ be another random sample from another population $N\left(\mu_{2}, \sigma^{2}\right)$. These two normal populations are known to have the same population variance, whose value $\sigma^{2}$ is however unknown to us, but they have not necessarily the same population mean. Under this situation the following statistical procedure is very often used by practical statistician: if $s_{2}^{2}$, the sample variance defined by $O_{n_{2}}$, is larger than a prescribed constant $C$, the sample mean $\bar{x}_{2}$ obtained from $O_{n_{2}}$ will not be so reliable as to be used alone without any regards to the stable information $\bar{x}_{1}$, and hence we may and we shall define the pooled estimate $\left(\boldsymbol{n}_{1} \bar{x}_{1}+\right.$ $\left.n_{2} \bar{x}_{2}\right) /\left(n_{1}+n_{2}\right)$, while otherwise, that is, if $s_{2}^{2}$ is equal to or smaller than the constant $C$, the sample mean $\bar{x}_{2}$ will be worth while to be used alone with no regards to the stable information $\bar{x}_{1}$.

The needs for such statistical procedure will occur when any increase of sample size $n_{2}$ is strictly prohibited or impossible and hence there is no way but we decide whether our information due to $O_{n_{2}}$ can be accepted or should be wholly abandoned.

Our statistical procedure is now formulated by defing a statistic $\bar{x}$ in the following way:

$$
\begin{array}{ll}
\text { (a) } \overline{\bar{x}}=\left(n_{1} \bar{x}_{1}+n_{2} \bar{x}_{2}\right) /\left(n_{1}+n_{2}\right), & \text { if } s_{2}^{2} \geq C, \\
\text { (b) } \overline{\bar{x}}=\bar{x}_{2}, & \text { if } s_{2}^{2}<C,
\end{array}
$$

where $C$ is a prescribed non-negative constant.

Theorem 1.1. The distribution function of $\bar{x}$ is given by

$$
\begin{aligned}
& \operatorname{Pr} .\{\bar{x}<u\}=\Phi\left(\frac{\left(n_{1}+n_{2}\right)}{1} \frac{u-\left(n_{1} \mu_{1}+n_{2} \mu_{2}\right)}{\sqrt[n_{1}+n_{2}]{ } \sigma}\right) \operatorname{Pr} .\left\{D_{1}\right\} \\
& +\Phi\left(\frac{\boldsymbol{n}_{1}\left(u-\mu_{1}\right)}{\sigma}\right) \cdot\left\{1-\operatorname{Pr} .\left\{D_{1}\right\}\right\},
\end{aligned}
$$

where we put 
(1.2) $\operatorname{Pr}\left\{D_{1}\right\}=\frac{1}{2 \Gamma\left(\frac{\boldsymbol{n}_{2}-1}{2}\right)} \int_{\frac{n_{2}}{\sigma^{2}} c}^{\infty}\left(\begin{array}{c}\chi^{2} \\ 2\end{array}\right)^{n_{2}-1} e^{-\frac{\chi^{2}}{2}} d \chi^{2}$,

and

(1.3) $\Phi(u)=\frac{1}{\mathrm{v} 2 \pi} \int_{-\infty}^{u} \exp \left\{-\frac{t^{2}}{2}\right\} d t$

while $D_{1}$ is the domain defined in the sample space $\left(x_{21}, x_{22}, \cdots, x_{2_{2}}\right)$ by the relation $s_{2}^{2}>C$ and $D_{2}$ is the complementary set of $D_{1}$ in the sample space.

Proof: This is easily shown from the fact that $s_{1}^{2}$ and $\left(\bar{x}_{1}, \bar{x}_{2}\right)$ are independently distributed. Indeed we have

$$
\operatorname{Pr} .\{\bar{x}<u\}=\operatorname{Pr} .\left\{\frac{n_{1} \bar{x}_{1}+n_{2} \bar{x}_{2}}{n_{1}+n_{2}}<u, D_{1}\right\}+\operatorname{Pr} .\left\{\bar{x}_{2}<u, D_{2}\right\},
$$

with $\operatorname{Pr} .\left\{D_{2}\right\}=1-\operatorname{Pr} .\left\{D_{1}\right\}$.

Theorem 1.2 The mean value $E\{\bar{x}\}$ and the mean square error M.S.E. $\{\overline{\bar{x}}\}$ of the estimate $\overline{\bar{x}}$ are given respectively

$$
E\{\overline{\bar{x}}\}=\mu_{2}+\frac{n_{2}}{n_{1}+n_{2}}\left(\mu_{1}-\mu_{2}\right) \cdot \operatorname{Pr} .\left\{D_{1}\right\}
$$

and

$$
\text { M.S.E. }\{\bar{x}\}=\frac{\sigma^{2}}{n_{2}}+\frac{n_{1}^{2} \sigma^{2}}{\left(n_{1}+n_{2}\right)^{2}} \cdot \operatorname{Pr} .\left\{D_{1}\right\} \cdot\left\{\frac{\left(\mu_{2}-\mu_{1}\right)^{2}}{\sigma^{2}}-\frac{\left(n_{1}+n_{2}\right)}{n_{1} n_{2}}\right\}
$$

Proof : (1.1) gives us directly (1.5), and (1.6) can be derived from

$$
E\left\{\overline{\boldsymbol{x}}^{2}\right\}=\left\{\frac{\sigma^{2}}{\boldsymbol{n}_{1}+\boldsymbol{n}_{2}}+\left(\begin{array}{c}
\boldsymbol{n}_{1} \mu_{1}+\boldsymbol{n}_{2} \mu_{2} \\
\boldsymbol{n}_{1}+\boldsymbol{n}_{2}
\end{array}\right)^{2}\right\} \cdot \operatorname{Pr} .\left\{D_{1}\right\}+\left(\frac{\sigma^{2}}{\boldsymbol{n}_{2}}+\mu_{2}^{2}\right) \cdot \operatorname{Pr} .\left\{D_{2}\right\} \text {. }
$$

\subsection{Type 2: (The inference of population mean after one-sided $t$-test)}

Let $O_{n_{1}}:\left(x_{11}, x_{12}, \cdots, x_{1 n_{1}}\right)$ be a random sample of $n_{1}$ from a normal population $N\left(\mu_{1}, \sigma^{2}\right)$, and let $O_{n_{2}}:\left(x_{21}, x_{22}, \cdots, x_{2 n_{2}}\right)$ be another random sample of $n_{2}$ drawn from another normal population $N\left(\mu_{2}, \sigma^{2}\right)$. These two normal populations are known to have the same population variance $\sigma^{2}$, whose value is however unknown to us. The distinction between two population means may be regarded as hypothetical and we are in the situation of an incompletely specified model in the sense of Bozivich-Bancroft-Hartley [1].

We are now concerned with the rule of the statistical procedure for- 
mulated in the following way:

(i) Let $\bar{x}_{i}$ and $s_{i}^{2}$ be the sample mean and the sample variance defined by $O_{n_{i}}(i=1,2)$.

(ii) Let the statistic $t$ be defined by

$$
t=\left(\bar{x}_{1}-\bar{x}_{2}\right) /\left\{\left(\frac{1}{n_{1}}+\frac{1}{n_{2}}\right)^{1 / 2}\left(\frac{n_{1} s_{1}^{2}+n_{2} s_{2}^{2}}{n_{1}+n_{2}-2}\right)^{1 / 2}\right\} .
$$

(iii) Let us define the statistic $\overline{\bar{x}}$ in the following way:

(a) $\overline{\bar{x}}=\bar{x}_{2}$, if $t \leqq \lambda$,

(b) $\overline{\bar{x}}=\left(n_{1} \bar{x}_{1}+n_{2} \bar{x}_{2}\right) /\left(n_{1}+n_{2}\right)$, if $\lambda<t<A$,

(c) $\overline{\bar{x}}=\bar{x}_{1}$, if $t \geqq \Lambda$,

where the switching constants $A$ and $\lambda$ are prescribed constants.

There are two important practical problems $\left(1^{\circ}\right)$ and $\left(2^{\circ}\right)$ to which our present statistical procedure can be applied with certain respective different interpretation.

$\left(1^{\circ}\right)$ The first problem is concerned with an estimation of maximum value of $\mu_{1}$ and $\mu_{2}$. The simplest estimate which can be verified by the theory of statistical decision function is known to be $\max \left(\bar{x}_{1}, \bar{x}_{2}\right)$, as shown in Bahadur [1]. Our inference procedure, however, adopts a modified estimate in order to aim at the improvement of the precision of an estimate of common population mean under the situation $\mu_{1}=\mu_{2}$.

$\left(2^{\circ}\right)$ The second problem is based upon assumption that $\mu_{1} \geqq \mu_{2}$. For instance this assumption may be quite reasonable when the second population is concernd with the observations on a control, while the first one with a manifestly more reinforced drug than the control, which involves $\mu_{1} \geq \mu_{2}$. Under this situation where the assumption $\mu_{1} \geq \mu_{2}$ hold true, an information which is given by two sample means, $\bar{x}_{1}$ and $\bar{x}_{2}$, when $\bar{x}_{1}$ happens to be significantly smaller than $\bar{x}_{2}$ can be admitted under the hypothesis that $\mu_{1}=\mu_{2}$ with the interpretation that $O_{n_{1}}$ is giving exceptionally small sample mean $\bar{x}_{1}$. Therefore we may and we shall in this case reject the information giving by $O_{n_{1}}$.

Theorem 2.1. The distribution function of $\bar{x}$ is given by

$$
\begin{aligned}
& \operatorname{Pr} .\{\overline{\bar{x}}<u\} \\
& =\frac{1}{V 2 \pi} \iint_{T \leq \lambda} e^{-\frac{p^{2}}{2}} \frac{V^{\frac{n_{1}+n_{2}}{2}-2} e^{-V}}{\Gamma\left(\frac{n_{1}+n_{2}-2}{2}\right)} \Phi\left(\frac{u-\mu_{2}}{\sigma} \sqrt{n_{1}+n_{2}}-\sqrt{\left.\frac{n_{1}}{n_{2}} p\right)} d p d V\right. \\
& \quad+\frac{1}{\sqrt{2 \pi}}=\left(\frac{\left(n_{1}+n_{2}\right)}{\sigma_{1}} \frac{u-\left(n_{1} \mu_{1}+n_{2} \mu_{2}\right)}{n_{1}+n_{2}}\right) \iint_{\lambda<T<\Lambda} e^{-\frac{p^{2}}{2}} \frac{V^{\frac{n_{1}+n_{2}}{2}-2} e^{-V}}{\Gamma\left(\frac{n_{1}+n_{2}-2}{2}\right)} d p d V
\end{aligned}
$$




$$
+\frac{1}{\imath<2 \pi} \int_{T \geq \Lambda} \int^{-\frac{p_{2}}{2}} \frac{V^{\frac{m_{1}+n_{2}}{2}-2} e^{-V}}{\Gamma\left(\frac{n_{1}+n_{2}-2}{2}\right)} \Phi\left(\frac{u-\mu_{1}}{\sigma} V / n_{1}+n_{2}+\sqrt{\frac{n_{2}}{n_{1}} p}\right) d p d V,
$$

where we put

$$
T \equiv\left(\sqrt{\frac{n_{1} n_{2}}{n_{1}+n_{2}}} \frac{\mu_{2}-\mu_{1}}{\sigma}+p\right) / \sqrt{\frac{2}{n_{1}+n_{2}-2} V} .
$$

Proof: The proof can be done along the same line as that to Theorem 1.1. explained in details in Kitagawa [2].

Theorem 2.2. The mean value $E\{\bar{x}\}$ and the mean square error M.S.E. $\{\bar{x}\}$ of estimate $\overline{\bar{x}}$ are given by

$$
\begin{gathered}
E\{\bar{x}\}=\mu_{1}-\frac{\left(\mu_{1}-\mu_{2}\right)}{n_{1}+n_{2}}\left\{n_{1} \operatorname{Pr} .\left\{D_{0}\right\}+n_{2}\left(1-\operatorname{Pr} .\left\{D_{2}\right\}\right)\right\} \\
+\frac{\sigma}{V n_{1}+n_{2}} \cdot\left\{\sqrt{\frac{n_{1}}{n_{2}}} I_{D_{0}}(p)-\sqrt{\frac{n_{2}}{n_{1}}} I_{L_{2}}(p)\right\}, \\
=\frac{n_{1} \mu_{1}+n_{2} \mu_{2}}{n_{1}+n_{2}}+\frac{\left(\mu_{1}-\mu_{2}\right)}{n_{1}+n_{2}}\left\{n_{2} \operatorname{Pr} .\left\{D_{2}\right\}-n_{1} \operatorname{Pr} .\left\{D_{0}\right\}\right\} \\
-\frac{\sigma}{\sqrt{n_{1} n_{2}\left(n_{1}+n_{2}\right)}}=\left\{n_{2} I_{\Gamma_{2}}(p)-n_{1} I_{D_{0}}(p)\right\},
\end{gathered}
$$

and

$$
\begin{gathered}
\text { M.S.E. }\{\bar{x}\}=\frac{\sigma^{2}}{n_{1}+n_{2}}+\frac{\sigma^{2}}{n_{1}+n_{2}}\left\{\frac{n_{2}}{n_{1}} I_{D_{2}}\left(p^{2}\right)+\frac{n_{1}}{n_{2}} I_{D_{0}}\left(p^{2}\right)\right\}+\left\{1-\operatorname{Pr} .\left\{D_{2}\right\}\right\} \\
\cdot\left(\frac{n_{2}\left(\mu_{1}-\mu_{2}\right)}{n_{1}+n_{2}}\right)^{2}+\operatorname{Pr} .\left\{D_{0}\right\} \cdot\left\{\mu_{1}+\frac{n_{2} \mu_{1}+n_{1} \mu_{2}}{n_{1}+n_{2}}\right\}\left\{\mu_{2}+\frac{n_{1} \mu_{1}+n_{2} \mu_{2}}{n_{1}+n_{2}}\right\},
\end{gathered}
$$

where we put

$$
\begin{aligned}
& I_{D_{i}}\left(p^{j}\right)=\iint_{D_{i}} p^{j} f(p, V) d p d V, \quad(j=1,2), \\
& f(p, V)=\frac{1}{\sqrt{2 \pi}} e^{-\frac{p_{2}^{2}}{2}} \frac{V^{\frac{n_{1}+n_{2}-2}{2} e^{-V}}}{\Gamma\left(\frac{n_{1}+n_{2}-2}{2}\right)}, \\
& D_{i}:\left\{\begin{array}{l}
T \leq \lambda,(i=0), \\
T \geq \Lambda,(i=2),
\end{array}\right.
\end{aligned}
$$

and 


$$
\operatorname{Pr} .\left\{D_{i}\right\}=\iint_{D_{i}} f(p, V) d p d V \text {. }
$$

Proof: The mean value $E\{\bar{x}\}$ is given by

$$
\begin{aligned}
& E\{\overline{\bar{x}}\}=\int_{-\infty}^{\infty}\left[\iint_{D_{1}}\left(\frac{n_{1} \mu_{1}+n_{2} \mu_{2}}{n_{1}+n_{2}}+\frac{\sigma q}{\sqrt{n_{1}+n_{2}}}\right) \frac{e^{-\frac{p^{2}+q^{2}}{2}-r} V^{\frac{n_{1}+n_{2}}{2}-2}}{2 \pi \Gamma\left(\frac{n_{1}+n_{2}-2}{2}\right)} d p d V\right.
\end{aligned}
$$

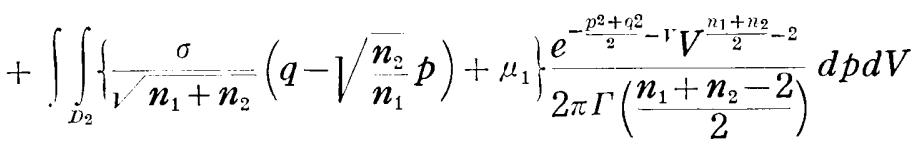

$$
\begin{aligned}
& +\iint_{D_{0}}\left\{\mu_{2}+\frac{\sigma}{\sqrt{n_{1}+n_{2}}}\left(q+\sqrt{\left.\frac{n_{1}}{n_{2}} p\right)} \frac{e^{-\frac{p^{2}+q^{2}}{2}-r} V^{n_{1}+n_{2}}-2}{2} d p d V\right] d q,\right.
\end{aligned}
$$

and the variance (2.5) is obtained from a following relation,

$$
\begin{aligned}
E\left\{\bar{x}^{2}\right\}= & \left\{\frac{\sigma^{2}}{n_{1}+n_{2}}+\left(\frac{n_{1} \mu_{1}+n_{2} \mu_{2}}{n_{1}+n_{2}}\right)^{2}\right\} \operatorname{Pr} .\left\{D_{1}\right\}+\left\{\frac{\sigma^{2}}{n_{1}+n_{2}}+\mu_{1}^{2}\right\} \cdot \operatorname{Pr} .\left\{D_{2}\right\} \\
& +\left\{\frac{\sigma^{2}}{n_{1}+n_{2}}+\mu_{2}^{2}\right\} \cdot \operatorname{Pr} .\left\{D_{0}\right\}+{ }_{n_{1}+n_{2}}{ }^{2}\left\{n_{1} I_{D_{0}}\left(p^{2}\right)+{ }_{n_{2}}^{n_{1}} I_{D_{2}}\left(p^{2}\right)\right\} \\
& +\frac{2 \sigma}{1 / n_{1}+n_{2}}\left\{\mu_{2} \sqrt{\frac{n_{1}}{n_{2}}} I_{D_{0}}(p)-\mu_{1} \sqrt{\frac{n_{2}}{n_{1}}} I_{D_{2}}(p)\right\} .
\end{aligned}
$$

Corollary 2.1. Specially when $\mu_{1}=\mu_{2}, \bar{x}$ is an unbiassed estimate of $\mu_{1}$, but its mean square error is greater than $\sigma^{2} /\left(n_{1}+n_{2}\right)$ and less than $\sigma^{2} / n_{1}$.

Corollary 2.2. Moreover if we put $\lambda=-\Lambda$, the results (2.1), (2.3) and (2.5) consist with the Theroem 1 in Kitagawa [1].

Corollary 2.3. Specially when $n_{1} \geq n_{2}$, the bias of $E\{\bar{x}\}$ becomes small and the mean square error becomes approximately equal to $\sigma^{2} /\left(n_{1}+n_{2}\right)$. Otherwise, when $n_{2} \gg n_{1}, E\{\overline{\bar{x}}\}$ is approximately equal to $\mu_{2}$, and the mean square error may become $\sigma^{2} I_{D_{2}}\left(p^{2}\right) / n_{1}$ nearly.

\subsection{Type 3: (The case with "known" population variances in Type 2)}

Let $O_{n_{1}}:\left(x_{11}, x_{12}, \cdots, x_{1_{1}}\right)$ be a random sample of $n_{1}$ from a normal population $N\left(\mu_{1}, \sigma_{1}^{2}\right)$, and let $O_{n_{2}}:\left(x_{21}, x_{22}, \cdots, x_{2 n_{2}}\right)$ be a random sample of $n_{2}$ from another normal population $N\left(\mu_{2}, \sigma_{2}^{2}\right)$. The values of these two population variances are known to us, but the populations have not necessarily the same population mean and variance. 
The rule of the statistical procedure is now specialized in the following way:

(i) Let $x_{i}$ be the sample mean defined from $O_{n_{i}}(i=1, \therefore)$.

(ii) Let the statistic $d$ be defined by $d=\left(\bar{x}_{1}-\bar{x}_{2}\right) \sqrt{\sigma_{\boldsymbol{n}_{1}}^{2}}+\frac{\sigma_{2}^{2}}{\bar{n}_{2}}$.

(iii) Let us define the statistic $x$ in the following way:

(a) $\bar{x}=x_{2}$, if $d \leq \lambda$,

(b) $\bar{x}=\left(n_{1} \bar{x}_{1}+n_{2} \bar{x}_{2}\right) /\left(n_{1}+n_{2}\right)$, if $\lambda<d<A$,

(c) $\overline{\bar{x}}=\bar{x}_{1}$, if $d \geq A$,

where the switching constants $\Lambda$ and $\lambda$ are prescribed constants.

We are now giving some observations on the mean and the variance of the statistic $\bar{x}$ with their detailed proofs, because the Type 3 is simpler case and suited for numerical considerations.

Theorem 3.1. The distribution function of $x$ is given by

$$
\begin{aligned}
& \operatorname{Pr} .\{\bar{x}<\boldsymbol{u}\}=\frac{V \bar{n}_{2}}{V \overline{2} \pi \sigma_{2}} \int_{-\infty}^{u-\mu_{2}}\left(\boldsymbol{y}+\mu_{2}\right) \Phi\left(\frac{\lambda+\frac{\mu_{2}-\mu_{1}}{c}+\frac{y}{c}}{V \frac{1-\rho_{1}^{2}}{2}}\right) e^{-\frac{n_{2} y^{2}}{2 \sigma_{22}}} d y
\end{aligned}
$$

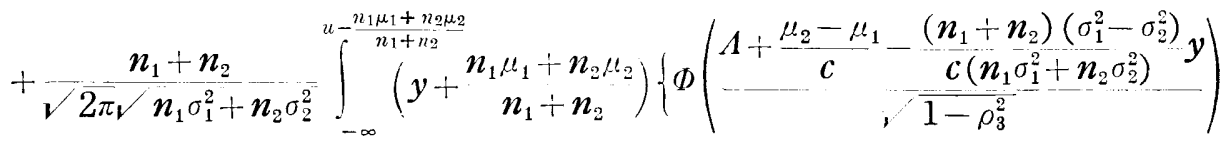

$$
\begin{aligned}
& \left.-\Phi\left(\frac{\lambda+\frac{\mu_{2}-\mu_{1}}{c}-\left(n_{1}+n_{2}\right)\left(\sigma_{1}^{2}-\sigma_{2}^{2}\right)}{c\left(\boldsymbol{n}_{1} \sigma+\boldsymbol{n}_{2} \sigma_{2}^{2}\right)} y\right)\right\} \times e^{-\frac{\left(n_{1}+\mu_{2}\right)^{2} y^{2}}{2\left(n_{1} \sigma_{1}+n_{2} \sigma_{2}\right)}} d y \\
& +\frac{v \overline{n_{1}}}{\nu} \int_{-\infty}^{u-\mu_{1}}\left(y+\mu_{1}\right)\left\{1-\Phi\left(\frac{A+\frac{\mu_{2}-\mu_{1}}{c \pi \sigma_{1}}-\frac{y}{c}}{V \frac{c}{1-\rho_{2}^{2}}}\right)\right\} e^{-\frac{u_{1} y^{2}}{2 \sigma_{1}^{2}}} d y \text {, }
\end{aligned}
$$

where $c, \rho_{1}, \rho_{2}$ and $\rho_{3}$ are defined by (3.4), (3.6) and (3.8).

Proof : Let us put

$$
\operatorname{Pr} .\{\bar{x}<u\}=\operatorname{Pr} .\left\{\bar{x}_{2}<u, D_{0}\right\}+\operatorname{Pr} .\left\{\frac{n_{1} x_{1}+n_{2} \bar{x}_{2}}{n_{1}+n_{2}}<u, D_{1}\right\}+\operatorname{Pr} .\left\{\bar{x}_{1}<u, D_{2}\right\},
$$

where $D_{0}, D_{1}$ and $D_{2}$ are the domains in the sample space $\left(x_{11}, \cdots, x_{1 n}, x_{21}\right.$, $\cdots, x_{2_{2}}$ ) defined by the above relations (a), (b) and (c) respectively. Then we have

$$
\begin{array}{r}
\operatorname{Pr} .\left\{x_{2}<u, D_{0}\right\}=\frac{1}{2 \pi \sigma_{X} \sigma_{Y} V} 1-\rho_{1}^{2} \int_{-\infty}^{u} Y\left\{\int _ { - \infty } ^ { \lambda } \operatorname { e x p } \left[\frac { - 1 } { 2 ( 1 - p _ { 1 } ^ { 2 } ) } \left[\frac{\left(X-\xi_{1}\right)^{2}}{\sigma_{X}^{2}}\right.\right.\right. \\
\left.\left.\left.-2 \rho_{1} \frac{\left(X-\xi_{1}\right)\left(Y-\xi_{2}\right)}{\sigma_{X} \sigma_{Y}}+\frac{\left(Y-\xi_{2}\right)^{2}}{\sigma_{Y}^{2}}\right]\right] d X\right\} d Y,
\end{array}
$$


where we put

$$
\left\{\begin{array}{l}
X=\frac{\overline{\boldsymbol{x}}_{1}-\boldsymbol{x}_{2}}{\boldsymbol{c}}, \quad Y=\boldsymbol{x}_{2}, \xi_{1}=\frac{\mu_{1}-\mu_{2}}{c}, \xi_{2}=\mu_{2}, \\
\sigma_{x}^{2}=1, \sigma_{Y}^{2}=\frac{\sigma_{2}^{2}}{\boldsymbol{n}_{2}}, \quad c=\sqrt{\frac{\sigma_{1}^{2}}{\boldsymbol{n}_{1}}+\frac{\sigma_{2}^{2}}{n_{2}}}, \\
\rho_{1}=-\frac{\sigma_{2}}{\boldsymbol{c}_{V}} \frac{\boldsymbol{n}_{2}}{\boldsymbol{n}_{2}} .
\end{array}\right.
$$

We have also

$$
\begin{aligned}
& \operatorname{Pr} .\left\{\frac{\boldsymbol{n}_{1} \overline{\boldsymbol{x}}_{1}+\boldsymbol{n}_{2} \overline{\boldsymbol{x}}_{2}}{\boldsymbol{n}_{1}+\boldsymbol{n}_{2}}<u, D_{1}\right\} \\
& \begin{aligned}
=\frac{1}{2 \pi \sigma_{X} \sigma_{T V} / 1-\rho_{3}^{2}} \int_{-\infty}^{u} T\left\{\int _ { \lambda } ^ { \Lambda } \operatorname { e x p } \left[\frac { - 1 } { 2 ( 1 - \rho _ { 3 } ^ { 2 } ) } \left[\frac{\left(X-\xi_{1}\right)^{2}}{\sigma_{X}^{2}}-2 \rho_{3} \frac{\left(X-\xi_{1}\right)\left(T-\xi_{3}\right)}{\sigma_{X} \sigma_{T}}\right.\right.\right. \\
\left.\left.\left.+\frac{\left(T-\xi_{3}\right)^{2}}{\sigma_{T}^{2}}\right]\right] d X\right\} d T,
\end{aligned}
\end{aligned}
$$

where we put

$$
\left\{\begin{array}{l}
\boldsymbol{T}=\frac{\boldsymbol{n}_{1} \overline{\boldsymbol{x}}_{1}+\boldsymbol{n}_{2} \overline{\boldsymbol{x}}_{2}}{\boldsymbol{n}_{1}+\boldsymbol{n}_{2}}, \quad \xi_{3}=\frac{\boldsymbol{n}_{1} \mu_{1}+\boldsymbol{n}_{2} \mu_{2}}{\boldsymbol{n}_{\mathbf{1}}+\boldsymbol{n}_{2}}, \quad \sigma_{T}^{2}=\frac{\boldsymbol{n}_{1} \sigma_{1}^{2}+\boldsymbol{n}_{2} \sigma_{2}^{2}}{\left(\boldsymbol{n}_{1}+\boldsymbol{n}_{2}\right)^{2}}, \\
\rho_{3}=\frac{-\sigma_{1}^{2}-\sigma_{2}^{2}}{\boldsymbol{c}_{\sqrt{\boldsymbol{n}_{1} \sigma_{1}^{2}+\boldsymbol{n}_{2} \sigma_{2}^{2}}}} .
\end{array}\right.
$$

Furthermore we have

$$
\begin{aligned}
& \operatorname{Pr} .\left\{\bar{x}_{1}<u, D_{2}\right\} \\
& \begin{aligned}
\frac{1}{2 \pi \sigma_{X} \sigma_{Z} / 1-\rho_{2}^{2}} \int_{-\infty}^{u} Z\left\{\int_{\Lambda}^{\infty} \exp \right. & {\left[\frac { - 1 } { 2 ( 1 - \rho _ { 2 } ^ { 2 } ) } \left[\frac{\left(X-\xi_{1}\right)^{2}}{\sigma_{X}^{2}}-2 \rho_{2} \frac{\left(X-\xi_{1}\right)\left(Z-\xi_{4}\right)}{\sigma_{X} \sigma_{Z}}\right.\right.} \\
& \left.\left.\left.+\frac{\left(Z-\xi_{4}\right)^{2}}{\sigma_{Z}^{2}}\right]\right] d X\right\} d Z,
\end{aligned}
\end{aligned}
$$

where we put

$$
\left\{\begin{array}{l}
Z=\bar{x}_{1}, \xi_{4}=\mu_{1}, \sigma_{z}^{2}=\frac{\sigma_{1}^{2}}{n_{1}}, \\
\rho_{2}=\frac{\sigma_{1}}{c_{V}} \cdot
\end{array}\right.
$$

(3.1) is now easily obtained from the changes of the order of integrations in each of the right-hand sides of (3.3), (3.5) and (3.7), as we were 
to prove.

Theorem 3.2. The mean value $E\{\bar{x}\}$ and the mean square error $E\{(\bar{x}-$ $\left.\left.\mu_{1}\right)^{2}\right\}$ of the estimate $\bar{x}$ are given by

(3.9) $E\{\overline{\bar{x}}\}=\mu_{1}+\left(\mu_{2}-\mu_{1}\right)\left\{\frac{n_{2}}{n_{1}+n_{2}} J_{1}\left(y^{0}\right)+J_{0}\left(y^{0}\right)\right\}+J_{0}\left(y^{1}\right)+J_{1}\left(y^{1}\right)-J_{2}\left(y^{1}\right)$,

and

$$
\begin{aligned}
& \text { M.S.E. }\{\bar{x}\}=\frac{\sigma_{1}^{2}}{n_{1}}+\left(\mu_{2}-\mu_{1}\right)^{2}\left\{J_{0}\left(y^{0}\right)+\frac{n_{2}^{2}}{\left(n_{1}+n_{2}\right)^{2}} J_{1}\left(y^{0}\right)\right\} \\
& \quad+2\left(\mu_{2}-\mu_{1}\right)\left\{J_{0}\left(y^{1}\right)-\frac{n_{2}}{n_{1}+n_{2}} J_{2}\left(y^{1}\right)\right\}+J_{0}\left(y^{2}\right)+J_{1}\left(y^{2}\right)-J_{2}\left(y^{2}\right),
\end{aligned}
$$

where we put

$$
\begin{aligned}
& \int J_{0}\left(y^{i}\right)=\frac{V n_{2}}{\sqrt{2 \pi \sigma_{2}}} \int_{-\infty}^{\infty} y^{i} \Phi\left(\frac{\lambda+\frac{\mu_{2}-\mu_{1}}{c}+\frac{y}{c}}{\sqrt{1-\rho_{1}^{2}}}\right) e^{-\frac{n_{2} y^{2}}{2 \sigma_{2} 2}} d y,
\end{aligned}
$$

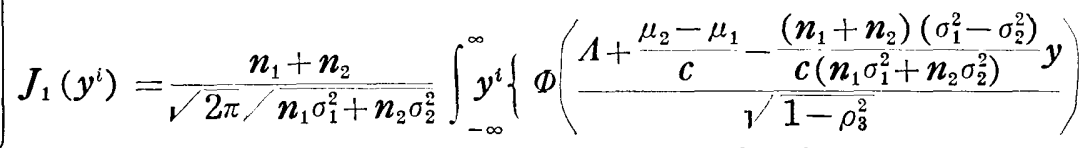

$$
\begin{aligned}
& \left\{-\Phi\left(\frac{\lambda+\frac{\mu_{2}-\mu_{1}}{c}-\frac{\left(n_{1}+n_{2}\right)\left(\sigma_{1}^{2}-\sigma_{2}^{2}\right)}{c\left(n_{1} \sigma_{1}^{2}+n_{2} \sigma_{2}^{2}\right)} y}{\sqrt{1-\rho_{3}^{2}}}\right)\right\} e^{-\frac{\left(n_{1}+n_{2}\right) 2 y^{2}}{2\left(n_{1} \sigma_{1}^{2}+n_{2} \sigma_{2} \sigma_{2}^{2}\right)}} d y, \\
& J_{2}\left(y^{i}\right)=\frac{\sqrt{n_{1}}}{\sqrt{2 \pi} \sigma_{1}} \int_{-\infty}^{\infty} y^{i} \Phi\left(\frac{\Lambda+\frac{\mu_{2}-\mu_{1}}{c}-\frac{y}{c}}{\sqrt{1-\rho_{2}^{2}}}\right) e^{-\frac{n_{1} y^{2}}{2 \sigma_{1}^{2}}} d y,
\end{aligned}
$$

for $i=0,1,2$.

Proof: The mean value (3.9) is evident, and the mean square error is obtained by

$$
\begin{aligned}
E\left\{\overline{\boldsymbol{x}}^{2}\right\}= & \frac{\sigma_{1}^{2}}{\boldsymbol{n}_{1}}+\mu_{1}^{2}+\left(\mu_{2}^{2}-\mu_{1}^{2}\right) J_{0}\left(\boldsymbol{y}^{0}\right)+\left\{\left(\frac{\boldsymbol{n}_{1} \mu_{1}+\boldsymbol{n}_{2} \mu_{2}}{\boldsymbol{n}_{1}+\boldsymbol{n}_{2}}\right)^{2}-\mu_{1}^{2}\right\} J_{1}\left(\boldsymbol{y}^{0}\right)+2 \mu_{2} J_{0}\left(\boldsymbol{y}^{1}\right) \\
& +2 \frac{\boldsymbol{n}_{1} \mu_{1}+\boldsymbol{n}_{2} \mu_{2}}{\boldsymbol{n}_{1}+\boldsymbol{n}_{2}} J_{1}\left(\boldsymbol{y}^{1}\right)-2 \mu_{1} J_{2}\left(\boldsymbol{y}^{1}\right)+J_{0}\left(\boldsymbol{y}^{2}\right)+J_{1}\left(\boldsymbol{y}^{2}\right)-J_{2}\left(y^{2}\right)
\end{aligned}
$$

Corollary 3.1. Specially when $\sigma_{1}=\sigma_{2}=\sigma$, the values of $E\{\bar{x}\}$ and M.S.E. $\{\overline{\bar{x}}\}$ are shown as follows:

$$
\begin{aligned}
E\{\bar{x}\}= & \mu_{1}+\left(\mu_{2}-\mu_{1}\right)\left[\frac{n_{2}}{n_{1}+n_{2}}\left\{\Phi\left(\Lambda+\frac{\mu_{2}-\mu_{1}}{c}\right)-\Phi\left(\lambda+\frac{\mu_{2}-\mu_{1}}{c}\right)\right\}+J_{0}\left(y^{0}\right)\right] \\
& +J_{0}\left(y^{1}\right)-J_{2}\left(y^{1}\right)
\end{aligned}
$$

and 


$$
\begin{aligned}
& \text { M.S.E. }\{\boldsymbol{x}\}=\frac{\sigma^{2}}{\boldsymbol{n}_{1}}+\left\{\Phi\left(\Lambda+\frac{\mu_{2}-\mu_{1}}{c}\right)-\Phi\left(\lambda+\frac{\mu_{2}-\mu_{1}}{c}\right)\right\} \boldsymbol{\sigma}_{1}+\boldsymbol{n}_{2} \\
& \quad+\left(\mu_{2}-\mu_{1}\right)^{2}\left[J_{0}\left(y^{0}\right)+\frac{\boldsymbol{n}_{2}^{2}}{\left(n_{1}+\boldsymbol{n}_{2}\right)^{2}}\left\{\Phi\left(\Lambda+\frac{\mu_{2}-\mu_{1}}{c}\right)-\Phi\left(\lambda+\frac{\mu_{2}-\mu_{1}}{c}\right)\right\}\right] \\
& \quad+2\left(\mu_{2}-\mu_{1}\right)\left\{J_{0}\left(y^{1}\right)-\frac{n_{2}}{n_{1}+n_{2}} J_{2}\left(y^{1}\right)\right\}+J_{0}\left(y^{2}\right)-J_{2}\left(y^{2}\right) .
\end{aligned}
$$

Corollary 3.2. Specially when $\mu_{1}=\mu_{2}, \bar{x}$ is an unbiased estimate of $\mu_{1}$ and M.S.E. $\{\overline{\bar{x}}\}$ becomes as follows:

$$
\text { M.S.E. }\{\overline{\boldsymbol{x}}\}=-\frac{\sigma_{1}^{2}}{n_{1}}+J_{0}\left(\boldsymbol{y}^{2}\right)+J_{1}\left(\boldsymbol{y}^{2}\right)-J_{2}\left(y^{2}\right),
$$

and in case when $\sigma_{1}=\sigma_{2}=\sigma$,

$$
\text { M.S.E. }\{\bar{x}\}=\frac{\sigma^{2}}{n_{1}}+\{\Phi(\Lambda)-\Phi(\lambda)\} \frac{\sigma^{2}}{n_{1}+n_{2}}+J_{0}\left(y^{2}\right)-J_{2}\left(y^{2}\right)
$$

Corollary 3.3. Specially when $\sigma_{1}^{2}=\sigma_{2}^{2}=\sigma^{2}$ and $n_{1} \gg n_{2}$, the bias of the estimate of $\overline{\bar{x}}$ becomes small and the mean square error becomes $\sigma^{2} /\left(n_{1}+n_{2}\right)$ approximately. Otherwise, when $n_{2} \gg n_{1}$, the estimate of $\overline{\bar{x}}$ becomes $\mu_{2}$ approximately.

\subsection{Type 4: (The inference of mean value after a preliminary test of variance)}

Let $O_{n_{1}}:\left(x_{11}, x_{12}, \cdots, x_{1 n_{1}}\right)$ be a random sample of $n_{1}$ from a normal population $N\left(\mu_{1}, \sigma_{1}^{2}\right)$ and let $O_{n_{2}}:\left(x_{21}, x_{22}, \cdots, x_{2 n_{2}}\right)$ be another random sample of $n_{2}$ from another normal population $N\left(\mu_{2}, \sigma_{2}^{2}\right)$. An equality of two population means is not necessarily assumed. However, if we can consider the values of these population means to be common, each of two sample means $\bar{x}_{1}$ and $\bar{x}_{2}$, can be used as an estimate of the common popuation mean $\mu\left(=\mu_{1}=\mu_{2}\right)$, and further at the same time the pooled sample mean $\bar{x}_{12}$ is also an estimate of $\mu$ with the variance $\sigma^{2}\left\{\overline{\boldsymbol{x}}_{12}\right\}=\left(\boldsymbol{n}_{1} \sigma_{1}^{2}+\boldsymbol{n}_{2} \sigma_{2}^{2}\right) /\left(\boldsymbol{n}_{1}+\boldsymbol{n}_{2}\right)^{2}$, which is greater than $\sigma_{1}^{2} / \boldsymbol{n}_{1}$ when $\sigma_{2}^{2} / \sigma_{1}^{2}<\left(2 n_{1}+n_{2}\right) n_{1}^{-1}$. This implies that the pooled sample mean $\bar{x}_{12}$ does not necessarily have a smaller variance than that of $x_{1}$.

Our rule of inference procedure of $\mu_{1}$ is formulated in the following way:

(i) Let $\bar{x}_{i}$ and $s_{i}^{2}$ be the sample mean and the sample unbiased variance defined from $O_{n_{i}}$, $(i=1,2)$.

(ii) Let the statistic $F$ be defined by $F=s_{2}^{2} / s_{1}^{2}$. 
(iii) Let us define the statistic $x$ in the following way:

(a) $\bar{x}=\bar{x}_{2}$, if $0<F \leq \lambda$,

(b) $\bar{x}=\left(n_{1} \bar{x}_{1}+n_{2} \bar{x}_{2}\right) /\left(n_{1}+n_{2}\right)$, if $\lambda<F<A$,

(c) $\overline{\bar{x}}=\bar{x}_{1}$, if $F \geq A$,

where the switching constants $A$ and $\lambda$ are prescribed constants.

There are two important practical problems $\left(1^{\circ}\right)$ and $\left(2^{\circ}\right)$ to which our present statistical inference procedure can be applied with their respective interpretation.

$\left(1^{\circ}\right)$ The first problem is concerned with the case when an equality of two population means can be assumed. Although the weighted mean $\left(\boldsymbol{n}_{1} \bar{x}_{1}\right.$ $\left.+\boldsymbol{n}_{2} \overline{\boldsymbol{x}}_{2}\right) /\left(\boldsymbol{n}_{1}+\boldsymbol{n}_{2}\right)$ is an unbiassed estimate of the common mean, our statistical practice based upon our common sense or upon our intuition will suggest us rather to reject a sample with significantly larger sample variances compared with the other one, and pool two sample means when two sample variances are not significantly different. In some biometrical and pharmaceutical researches, such a consideration concerning variances may be needed because of data containing a large fluctuation due to the individual subjects.

$\left(2^{\circ}\right)$ The second problem is concerned with an estimation of minimum value of $\mu_{1}$ and $\mu_{2}$ under the situation that population mean in consideration is a monotone increasing $g\left(\sigma^{2}\right)$ of its population variance $\sigma^{2}$. The function $g(\cdot)$ may or may not be known to us. It is therefore our intention for appealing to the statistical procedure of the Type 4 that a more reliable estimate of the common value $\mu_{1}=\mu_{2}$ can be obtained for the case of equal variance $\sigma_{1}^{2}=\sigma_{2}^{2}$ by pooling two sample means $\bar{x}_{1}$ and $\bar{x}_{2}$.

Theorem 4.1. The distribution function of $\bar{x}$ is given by

$$
\begin{gathered}
\operatorname{Pr} .\{\bar{x}<u\}=\Phi\left(\frac{1-n_{2}\left(u-\mu_{2}\right)}{\sigma_{2}}\right)\left\{1-I_{\theta_{0}}\left(\frac{\phi_{1}}{2}, \frac{\phi_{2}}{2}\right)\right\}+\Phi\left(\frac{\left(n_{1}+n_{2}\right) u-\left(n_{1} \mu_{1}+n_{2} \mu_{2}\right)}{V n_{1} \sigma_{1}^{2}+n_{2} \sigma_{2}^{2}}\right) \\
\cdot\left\{I_{\theta_{0}}\left(\frac{\phi_{1}}{2}, \frac{\phi_{2}}{2}\right)-I_{\theta_{2}}\left(\begin{array}{c}
\phi_{1} \\
2
\end{array}, \frac{\phi_{2}}{2}\right)\right\}+\Phi\left(\frac{V \overline{n_{1}}\left(u-\mu_{1}\right)}{\sigma_{1}}\right) I_{\theta_{2}}\left(\frac{\phi_{1}}{2}, \frac{\phi_{2}}{2}\right),
\end{gathered}
$$

where we put

$$
\begin{gathered}
\boldsymbol{I}_{\theta}\left(\begin{array}{c}
\phi_{1} \\
2
\end{array}, \frac{\phi_{2}}{2}\right)=\boldsymbol{B}_{\theta}\left(\begin{array}{c}
\phi_{1} \\
2
\end{array}, \frac{\phi_{2}}{2}\right) / \boldsymbol{B}\left(\frac{\phi_{1}}{2}, \frac{\phi_{2}}{2}\right), \phi_{1}=n_{1}-1, \phi_{2}=\boldsymbol{n}_{2}-1, \\
\theta_{0}=\phi_{2} /\left(\phi_{1} \lambda+\phi_{2}\right), \theta_{2}=\phi_{2} /\left(\phi_{1} \Lambda+\phi_{2}\right),
\end{gathered}
$$

and $B\left(\phi_{1} / 2, \phi_{2} / 2\right)$ and $B_{\theta}\left(\phi_{1} / 2, \phi_{2} / 2\right)$ denote a complete and an incomplete beta-function respectively.

Proof : This result is evident, because $F$ and $\bar{x}$ are independent of each other.

Theorem 4.2. The mean value $E\{\bar{x}\}$ and the mean square error $E\{(\overline{\bar{x}}$ $\left.\left.-\mu_{1}\right)^{2}\right\}$ are given by 


$$
E\{\overline{\bar{x}}\}=\mu_{2}+\frac{\left(\mu_{1}-\mu_{2}\right)}{n_{1}+n_{2}}\left\{n_{1} I_{\theta_{0}}\left(\frac{\phi_{1}}{2}, \frac{\phi_{2}}{2}\right)+n_{2} I_{\theta_{2}}\left(\frac{\phi_{1}}{2}, \frac{\phi_{2}}{2}\right)\right\},
$$

and

$$
\begin{aligned}
& E\left\{\left(\bar{x}-\mu_{1}\right)^{2}\right\}=\left(\mu_{1}-\mu_{2}\right)^{2}+\frac{\sigma_{2}^{2}}{\boldsymbol{n}_{2}}+\left\{\frac{\boldsymbol{n}_{1} \sigma_{1}^{2}+\boldsymbol{n}_{2} \sigma_{2}^{2}}{\left(\boldsymbol{n}_{\mathbf{1}}+\boldsymbol{n}_{2}\right)^{2}}-\frac{\sigma_{2}^{2}}{\boldsymbol{n}_{2}}-\frac{\boldsymbol{n}_{1}\left(\boldsymbol{n}_{1}+2 \boldsymbol{n}_{2}\right)\left(\mu_{1}-\mu_{2}\right)^{2}}{\left(\boldsymbol{n}_{\mathbf{1}}+\boldsymbol{n}_{2}\right)^{2}}-\right. \\
& \cdot \boldsymbol{I}_{\theta_{0}}\left(\frac{\phi_{1}}{2}, \frac{\phi_{2}}{2}\right)+\left\{\frac{\sigma_{1}^{2}}{\boldsymbol{n}_{1}}-\frac{\boldsymbol{n}_{1} \sigma_{1}^{2}+\boldsymbol{n}_{2} \sigma_{2}^{2}}{\left(\boldsymbol{n}_{1}+\boldsymbol{n}_{2}\right)^{2}}-\frac{\boldsymbol{n}_{2}^{2}\left(\mu_{1}-\mu_{2}\right)^{2}}{\left(\boldsymbol{n}_{1}+\boldsymbol{n}_{2}\right)^{2}}\right\} \boldsymbol{I}_{\theta_{2}}\left(\frac{\phi_{1}}{2}, \frac{\phi_{2}}{2}\right),
\end{aligned}
$$

where $\mu_{12}=\left(\boldsymbol{n}_{1} \mu_{1}+\boldsymbol{n}_{2} \mu_{2}\right) /\left(\boldsymbol{n}_{1}+\boldsymbol{n}_{2}\right)$.

Proof: The mean (4.3) is evident and M.S.E. $\{\overline{\bar{x}}\}$ is obtained from that

$$
\begin{aligned}
\boldsymbol{E}\left\{\overline{\boldsymbol{x}}^{2}\right\}= & \left(\frac{\sigma_{2}^{2}}{\boldsymbol{n}_{2}}+\mu_{2}^{2}\right)\left\{1-\boldsymbol{I}_{\theta_{0}}\left(\frac{\phi_{1}}{2}, \frac{\phi_{2}}{2}\right)\right\} \\
& +\left(\frac{\boldsymbol{n}_{1} \sigma_{1}^{2}+\boldsymbol{n}_{2} \sigma_{2}^{2}}{\left(\boldsymbol{n}_{1}+\boldsymbol{n}_{2}\right)^{2}}+\mu_{12}^{2}\right)\left\{\boldsymbol{I}_{\theta_{0}}\left(\frac{\phi_{1}}{2}, \frac{\phi_{2}}{2}\right)-I_{\theta_{2}}\left(\frac{\phi_{1}}{2}, \frac{\phi_{2}}{2}\right)\right\}+\left(\frac{\sigma_{1}^{2}}{\boldsymbol{n}_{1}}+\mu_{1}^{2}\right) I_{\theta_{2}}\left(\frac{\phi_{1}}{2}, \frac{\phi_{2}}{2}\right) .
\end{aligned}
$$

Corollary 4.1. Specially when $\mu_{1}=\mu_{2}, \overline{\bar{x}}$ is an unbiassed estimate of $\mu_{1}$ and M.S.E. $\{\bar{x}\}$ becomes as follows:

$$
\text { M.S.E. }\{\overline{\boldsymbol{x}}\}=\frac{\sigma_{2}^{2}}{\boldsymbol{n}_{2}}+\left(\frac{\boldsymbol{n}_{1} \sigma_{1}^{2}+\boldsymbol{n}_{2} \sigma_{2}^{2}}{\left(\boldsymbol{n}_{1}+\boldsymbol{n}_{2}\right)^{2}}-\frac{\sigma_{2}^{2}}{\boldsymbol{n}_{2}}\right) I_{\theta_{0}}\left(\frac{\phi_{1}}{2}, \frac{\phi_{2}}{2}\right)+\left(\frac{\sigma_{1}^{2}}{\boldsymbol{n}_{1}}-\frac{\boldsymbol{n}_{1} \sigma_{1}^{2}+\boldsymbol{n}_{2} \sigma_{2}^{2}}{\left(\boldsymbol{n}_{1}+\boldsymbol{n}_{2}\right)^{2}}\right) I_{\theta_{2}}\left(\frac{\phi_{1}}{2}, \frac{\phi_{2}}{2}\right) \text {. }
$$

Corollary 4.2. Moreover if we put $\lambda=0, E\{\overline{\bar{x}}\}$ and M.S.E. $\{\bar{x}\}$ are given by

$$
E\{\overline{\boldsymbol{x}}\}=\mu_{12}+\frac{\mu_{1}-\mu_{2}}{\boldsymbol{n}_{1}+\boldsymbol{n}_{2}} \boldsymbol{n}_{2} I_{\theta_{2}}\left(\frac{\phi_{1}}{2}, \frac{\phi_{2}}{2}\right),
$$

and

(4.8) M.S.E. $\{\ddot{\boldsymbol{x}}\}=\frac{\boldsymbol{n}_{2}^{2}\left(\mu_{1}-\mu_{2}\right)^{2}}{\left(\boldsymbol{n}_{1}+\boldsymbol{n}_{2}\right)^{2}}+\frac{\boldsymbol{n}_{1} \sigma_{1}^{2}+\boldsymbol{n}_{2} \sigma_{2}^{2}}{\left(\boldsymbol{n}_{1}+\boldsymbol{n}_{2}\right)^{2}}$

$$
+\left(\frac{\sigma_{1}^{2}}{\boldsymbol{n}_{1}}-\frac{\boldsymbol{n}_{1} \sigma_{1}^{2}+\boldsymbol{n}_{2} \sigma_{2}^{2}}{\left(\boldsymbol{n}_{1}+\boldsymbol{n}_{2}\right)^{2}}-\frac{\boldsymbol{n}_{2}^{2}\left(\mu_{1}-\mu_{2}\right)^{2}}{\left(\boldsymbol{n}_{1}+\boldsymbol{n}_{2}\right)^{2}}\right) \boldsymbol{I}_{\theta_{2}}\left(\frac{\phi_{1}}{2}, \frac{\phi_{2}}{2}\right) \text {. }
$$

Corollary 4.3. Specially the inference procedure defined by always pooling shows us that $E\{\bar{x}\}=\mu_{12}$ and M.S.E. $\{\bar{x}\}=\frac{n_{2}^{2}\left(\mu_{1}-\mu_{2}\right)^{2}}{\left(n_{1}+n_{2}\right)^{2}}+\frac{n_{1} \sigma_{1}^{2}+n_{2} \sigma_{2}^{2}}{\left(n_{1}+n_{2}\right)^{2}}$, while that defined by never-pooling, that is, $\lambda=\Lambda$, shows us that $E\{\bar{x}\}=$ $\mu_{2}+\left(\mu_{1}-\mu_{2}\right) I_{\theta}\left(\phi_{1} / 2, \phi_{2} / 2\right)$ and M.S.E. $\{\overline{\boldsymbol{x}}\}=\left(\mu_{1}-\mu_{2}\right)^{2}+\frac{\sigma_{2}^{2}}{n_{2}}+\left(\frac{\sigma_{1}^{2}}{n_{1}}-\frac{\sigma_{2}^{2}}{n_{2}}-\left(\mu_{1}-\mu_{2}\right)^{2}\right)$ 
$\cdot I_{\theta}\left(\frac{\phi_{1}}{2}, \frac{\phi_{2}}{2}\right)$, where $\theta=\theta_{0}=\theta_{2}$.

\section{§. Inference about population variances}

\subsection{Type 5: (The inference of a parameter after a preliminary test of analysis of variance)}

Let us consider an ordinary inference in case of a randomized block design as a fundamental example of analysis of variance test. Let $x_{i j}$ be a random sample drawn from $N\left(\theta_{. .}+\theta_{i .}+\theta_{\cdot j}, \sigma^{2}\right),(i=1,2, \cdots, l$ and $j=1,2, \cdots$, $m$ ), where $\theta_{i}$. and $\theta_{\cdot j}$ denote a treatment effect and a block effect respectively, and where we put $\sum_{i} \theta_{i .}=\sum_{j} \theta_{._{j}}=0$. Then the treatment effects are tested by the ratio $F_{0}$, that is,

$$
F_{0}=\frac{Q_{1}}{(l-1)} / \frac{Q_{3}}{(l-1)(m-1)},
$$

where $Q_{1}=m \sum_{i}\left(\bar{x}_{i \cdot}-\bar{x}_{. .}\right)^{2}$ and $Q_{3}=\sum_{i} \sum_{j}\left(x_{i j}-\bar{x}_{i \cdot}-\bar{x}_{._{j}}+\bar{x}_{. .}\right)^{2}$. The ratio $F_{0}$ is distributed in the central $F$-distribution with the pair $\{l-1,(l-1)(m-1)\}$ of the degrees of freedom under the null hypothesis $\theta_{i}=0$ for all $i$, and in the non-central $F$-distribution with the same pair of degree of freedom and with the parameter of the non-centricity parameter $\lambda=m \sum_{\imath} \theta_{i \cdot}^{2} / 2 \sigma^{2}$ under an alternative hypothesis.

We are now concerned with the statistical inference procedure of the parameter $\sum_{i} \theta_{i \cdot}^{2} /(l-1) \equiv \sigma_{t}^{2}$ defined in the following way:

(i) Let $F_{0}$ be a statistic defined by $F_{0}=\frac{Q_{1}}{(l-1)} / \frac{Q_{3}}{(l-1)(m-1)}$. way :

(ii) Let us introduce the estimate $\hat{\sigma}_{t}^{2}$ of $\sigma_{t}^{2}$ defined in the following

(a) $\hat{\sigma}_{t}^{2}=K$, if $F_{0}<A$,

(b) $\hat{\sigma}_{t}^{2}=\left(Q_{1}-\frac{Q_{3}}{m-1}\right) / m(l-1)$, if $F_{0} \geq \Lambda$,

where the switching $A$ and the estimate $K$ are certain prescribed non-negative constants.

There are two important practical problems $\left(1^{\circ}\right)$ and $\left(2^{\circ}\right)$ to which our present statistical inference procedure can be applied with their respective interpretation.

$\left(1^{\circ}\right)$ The first problem is concerned with an estimation of $\sigma_{t}^{2}$ under the situation when the assumption $\sigma_{t}^{2}>0$ is ascertained by an existence-proof on some physical grounds. Now let a non-significance of the $F$-test be infered 
from experiments, which might lead us to the conclusion to the effect $\sigma_{t}^{2}=0$. This is contradictory to our general and stable information to the effect that unless any additional and reliable data giving reasonable estimate of $\sigma_{t}^{2}$ are given, $\sigma_{t}^{2}$ should be better to be taken as equal to a certain non-negative constant $K$. Now we have obtained the data which is contradictory to our general assumption $\sigma_{t}^{2}>0$. Furthermore let it be prohibited or impossible to make further additional observations. Then our statistical procedure may be at least one of the possible approaches for estimating the constant $\sigma_{t}^{2}$.

$\left(2^{\circ}\right)$ The special case when $K=0$ may be worth while to mention, because, even in the case when no positive constant $K$ can be assumed in the sense enunciated in $\left(1^{\circ}\right)$, it may be natural (or operationally granted) at least for certain practical circumstances to assume that an estimate of $\sigma_{t}^{2}$ should not be negative. These considerations will hold valid, particularly for the case when any additional of size of sample is prohibited or impossible.

Theorem 5.1. The distribution function of $\hat{\sigma}_{t}^{2}$ is given by

$$
\begin{aligned}
& \operatorname{Pr} .\left\{\hat{\sigma}_{t}^{2}<u\right\}=1-\sum_{i=0}^{\infty} e^{-\lambda} \lambda^{i}\left[I_{\tau}\left(\frac{\nu_{1}}{2}+i, \frac{\nu_{2}}{2}\right)-\frac{\left(\frac{\nu_{2}}{\nu_{1}}\right)^{\frac{\nu_{2}}{2}}}{\left(2 \sigma^{2}\right)^{\frac{\nu_{1}+\frac{\nu_{2}}{2}+i}{2}} \Gamma\left(\frac{\nu_{1}}{2}+i ; \Gamma\left(\frac{\nu_{2}}{2}\right)\right.}\right.
\end{aligned}
$$

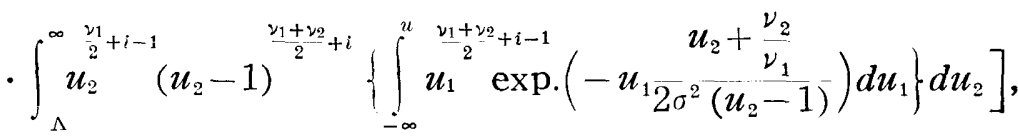

where $I_{\tau}\left(\begin{array}{c}\nu_{1} \\ 2\end{array}+i, \frac{\nu_{2}}{2}\right)$ is defined as same as (4.2), and

$$
\begin{aligned}
& \tau=\nu_{2} /\left(\nu_{1} A+\nu_{2}\right), \lambda=\sum_{i} \theta_{i \cdot}^{2} /(l-1), \\
& \nu_{1}=l-1, \nu_{2}=(l-1)(\boldsymbol{m}-1) .
\end{aligned}
$$

Proof : Let us put

$$
\operatorname{Pr} .\left\{\hat{\sigma}_{l}^{2}<u\right\}=\operatorname{Pr} .\left\{K, D_{(a)}\right\}+\operatorname{Pr} .\left\{\left(Q_{1}-\frac{Q_{3}}{m-1}\right) / m(l-1), D_{(b)}\right\}
$$

where $D_{(a)}$ is the domain defined in the sample space by the above relation (a) and $D_{(b)}$ is complement set of $D_{(a)}$ in the sample space. Now Pr. $\left\{K, D_{(a)}\right\}$ is a value of the definite integral ranging from zero to $A$ concerning probability density function of the non-central $F$-distribution with the non-centricity parameter $\lambda$ and the pair of the degrees of freedom $\{(l-1),(l-1)(m-1)\}$. The second term of the right side of (5.4) is calculated in the following way. Indeed the joint distribution of $Q_{1}$ and $Q_{3}$ is 


$$
\sum_{i=0}^{\infty} C_{i} Q_{3}{ }^{\nu_{2}-1} Q_{1}{ }^{{ }^{2}}{ }^{+i-1} \exp \left\{-\frac{1}{2}\left(\frac{Q_{3}+Q_{1}}{\sigma^{2}}\right)\right\} d Q_{1} d Q_{3}
$$

where

$$
C_{i} \equiv \frac{e^{-\lambda} \lambda^{i}}{\left(2 \sigma^{2}\right)^{\frac{\nu_{1}+\nu_{2}}{2}+i} i ! \Gamma\left(\frac{\nu_{2}}{2}\right) \Gamma\left(\frac{\nu_{1}}{2}+i\right)} .
$$

Now let us put $u_{1}=Q_{1}-\frac{\nu_{1}}{\nu_{2}} Q_{3}$ and $u_{2}=\nu_{2} Q_{1} / \nu_{1} Q_{3}$ and lit us transform $Q_{1}$ and $Q_{2}$ to $u_{1}$ and $u_{2}$. The Jacobian is given by

$$
\boldsymbol{J}=u_{1} \nu_{2} / \nu_{1}\left(u_{2}-1\right)^{2} .
$$

Consequently the right side of (5.2) is obtained.

Theorem 5.2. The mean value $E\left\{\hat{\sigma}_{t}^{2}\right\}$, the square mean $E\left\{\left(\hat{\sigma}_{t}^{2}\right)^{2}\right\}$ and the mean square error M.S.E. $\left\{\hat{\sigma}_{t}^{2}\right\}$ are given by

$$
\begin{aligned}
& E\left\{\hat{\sigma}_{t}^{2}\right\}=K \cdot\left\{1-\sum_{i=0}^{\infty} \frac{e^{-\lambda} \lambda^{i}}{i !} I_{\tau}\left(\sum_{2}^{\nu_{1}}+i, \frac{\nu_{2}}{2}\right)\right\}+\frac{2 \sigma^{2}}{\left(\nu_{1}+\nu_{2}\right)} \sum_{i=0}^{\infty} \frac{e^{-\lambda} \lambda^{i} \Gamma\left(\frac{\nu_{1}+\nu_{2}}{2}+i+1\right)}{i ! \Gamma\left(\frac{\nu_{2}}{2}\right) \Gamma\left(\frac{\nu_{1}}{2}+i\right)} \\
& \cdot\left[B\left(\frac{\nu_{1}}{2}+i+1, \frac{\nu_{2}}{2}\right)-B_{\phi^{\prime}}\left(\frac{\nu_{1}}{2}+i+1, \frac{\nu_{2}}{2}\right)-\frac{\nu_{1}}{\nu_{2}}\left\{B\left(\frac{\nu_{1}}{2}+i, \frac{\nu_{2}}{2}+1\right)\right.\right. \\
& \left.\left.-B_{\psi^{\prime}}\left(\frac{\nu_{1}}{2}+i, \frac{\nu_{2}}{2}+1\right)\right\}\right], \quad(\Lambda \geq 1), \\
& =K \cdot\left\{1-\sum_{i=0}^{\infty} \frac{e^{-\lambda} \lambda^{i}}{i !} I_{\tau}\left(\begin{array}{l}
\nu_{1} \\
2
\end{array}+i, \frac{\nu_{3}}{2}\right)\right\}+\frac{2 \sigma^{2}}{\left(\nu_{1}+\nu_{2}\right)} \sum_{i=0}^{\infty} \frac{e^{-\lambda} \lambda^{i} \Gamma\left(\frac{\nu_{1}+\nu_{2}}{2}+i+1\right)}{i ! \Gamma\left(\frac{\nu_{1}}{2}\right) \Gamma\left(\frac{\nu_{2}}{2}+i\right)} \\
& \cdot\left[B\left(\frac{\nu_{1}}{2}+i+1, \frac{\nu_{2}}{2}\right)-2 B_{\phi^{\prime \prime}}\left(\frac{\nu_{1}}{2}+i+1, \frac{\nu_{2}}{2}\right)-{ }_{\nu_{2}}^{\nu_{1}}\left\{B\left(\frac{\nu_{1}}{2}+i, \frac{\nu_{2}}{2}+1\right)\right.\right. \\
& \left.\left.-2 B_{\phi^{\prime \prime}}\left(\frac{\nu_{1}}{2}+i, \frac{\nu_{2}}{2}+1\right)+B_{\phi^{\prime}}\left(\frac{\nu_{1}}{2}+i, \frac{\nu_{2}}{2}+1\right)\right\}+B_{\phi^{\prime}}\left(\frac{\nu_{1}}{2}+i+1, \frac{\nu_{2}}{2}\right)\right], \\
& (\Lambda \leq 1) \text {, } \\
& E\left\{\left(\hat{\sigma}_{t}^{2}\right)^{2}\right\}=K^{2} \cdot\left\{1-\sum_{i} \frac{e^{-\lambda} \lambda^{i}}{i !} I_{\tau}\left(\frac{\nu_{1}}{2}+i, \frac{\nu_{2}}{2}\right)\right\}+\frac{\left(2 \sigma^{2}\right)^{2}}{\left(\nu_{1}+\nu_{2}\right)^{2}} \sum_{i} \frac{e^{-\lambda} \lambda^{i} \Gamma\left(\frac{\nu_{1}+\nu_{2}}{2}+i+2\right)}{i ! \Gamma\left(\frac{\nu_{2}}{2}\right) \Gamma\left(\frac{\nu_{1}}{2}+i\right)} \\
& \cdot\left[\nu_{2}\left\{B\left(\frac{\nu_{1}}{2}+i+2, \frac{\nu_{2}}{2}\right)-B_{\phi^{\prime}}\left(\frac{\nu_{1}}{2}+i+2, \frac{\nu_{2}}{2}\right)\right\}-2 \nu_{1}\left\{B\left(\begin{array}{c}
\nu_{1} \\
2
\end{array}+i+1, \frac{\nu_{2}}{2}+1\right)\right.\right.
\end{aligned}
$$




$$
\left.\left.-B_{\phi^{\prime}}\left(\frac{\nu_{1}}{2}+i+1, \frac{\nu_{2}}{2}+1\right)\right\}+\frac{\nu_{1}^{2}}{\nu_{2}}\left\{B\left(\frac{\nu_{1}}{2}+i, \frac{\nu_{2}}{2}+2\right)-B_{\phi^{\prime}}\left(\frac{\nu_{1}}{2}+i, \frac{\nu_{2}}{2}+2\right)\right\}\right]
$$

$(\Lambda \geq 1)$,

$$
\begin{aligned}
5.11)=K^{2} & \cdot\left\{1-\sum_{i} \frac{e^{-\lambda} \lambda^{i}}{i !} I_{\tau}\left(\frac{\nu_{1}}{2}+i, \frac{\nu_{2}}{2}\right)\right\}+\frac{\left(2 \sigma^{2}\right)^{2}}{\left(\nu_{1}+\nu_{2}\right)^{2}} \frac{e^{-\lambda} \lambda^{i} \Gamma\left(\frac{\nu_{1}+\nu_{2}}{2}+i+2\right)}{i ! \Gamma\left(\frac{\nu_{2}}{2}\right) \Gamma\left(\frac{\nu_{1}}{2}+i\right)} \\
\cdot & {\left[\nu_{2} B\left(\frac{\nu_{1}}{2}+i+2, \frac{\nu_{2}}{2}\right)-2 \nu_{1} B\left(\frac{\nu_{1}}{2}+i+1, \frac{\nu_{2}}{2}+1\right)+\frac{\nu_{1}^{2}}{\nu_{2}} B\left(\frac{\nu_{1}}{2}+i, \frac{\nu_{2}}{2}+2\right)\right.} \\
& -2\left\{\nu_{2} B_{\phi^{\prime \prime}}\left(\frac{\nu_{1}}{2}+i+2, \frac{\nu_{2}}{2}\right)-2 \nu_{1} B_{\phi^{\prime \prime}}\left(\frac{\nu_{1}}{2}+i+1, \frac{\nu_{2}}{2}+1\right)+\frac{\nu_{1}^{2}}{\nu_{2}} B_{\phi^{\prime \prime}}\left(\frac{\nu_{1}}{2}+i, \frac{\nu_{2}}{2}+2\right)\right\} \\
+ & \left.\nu_{2} B_{\phi^{\prime}}\left(\frac{\nu_{1}}{2}+i+2, \frac{\nu_{2}}{2}\right)-2 \nu_{1} B_{\phi^{\prime}}\left(\frac{\nu_{1}}{2}+i+1, \frac{\nu_{2}}{2}+1\right)+\frac{\nu_{1}^{2}}{\nu_{2}} B_{\phi^{\prime}}\left(\frac{\nu_{1}}{2}+i, \frac{\nu_{2}}{2}+2\right)\right],
\end{aligned}
$$

$(\Lambda \leq 1)$,

and

$$
\begin{aligned}
\text { M.S.E. }\left\{\hat{\sigma}_{t}^{2}\right\} & =E\left\{\left(\hat{\sigma}_{t}^{2}-\frac{\sum_{i} \theta_{i \cdot}^{2}}{\nu_{1}}\right)^{2}\right\}=E\left\{\left(\hat{\sigma}_{t}^{2}-\frac{2 \sigma^{2} \lambda}{\nu_{1}+\nu_{2}}\right)^{2}\right\} \\
& =E\left\{\hat{\sigma}_{t}^{4}\right\}-\frac{4 \cdot \sigma^{2} \lambda}{\nu_{1}+\nu_{2}} E\left\{\hat{\sigma}_{t}^{2}\right\}+\frac{4 \sigma^{2}}{\left(\nu_{1}+\nu_{2}\right)^{2}} \lambda^{2},
\end{aligned}
$$

where we put

$$
\begin{aligned}
& \tau=\nu_{2} /\left(\nu_{2}+\nu_{1} \Lambda\right), \nu_{1}=l-1, \nu_{2}=(l-1)(m-l), \\
& \phi^{\prime}=1-\tau, \quad \phi^{\prime \prime}=\nu_{1} /\left(\nu_{1}+\nu_{2}\right) .
\end{aligned}
$$

Proof: The mean value and the mean square value concerning to $\left(Q_{1}-\frac{\nu_{1}}{\nu_{2}} Q_{3}\right)$ are obtained in the following way:

$$
\begin{aligned}
& E\left\{Q_{1}-\frac{\nu_{1}}{\nu_{2}} Q_{3}, D_{(i)}\right\} \equiv \sum_{i} C_{i}\left(\frac{\nu_{2}}{\nu_{1}}\right)^{\frac{\nu_{2}}{2}}\left(2 \sigma^{2}\right)^{\frac{\nu_{1}+\nu_{2}}{2}+i+1} \Gamma\left(\frac{\nu_{1}+\nu_{2}}{2}+i+1\right)
\end{aligned}
$$

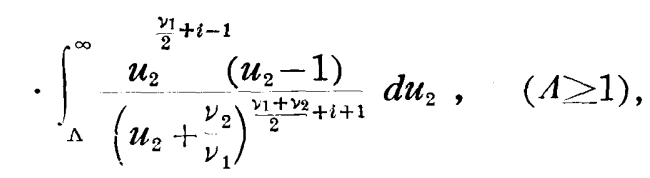

$$
\begin{aligned}
& =\sum_{i} C_{i}\left(\frac{\nu_{2}}{\nu_{1}}\right)^{\frac{\nu_{2}}{2}}\left(2 \sigma^{2}\right)^{\frac{\nu_{1}+\nu_{2}}{2}+i+1} \Gamma\left(\frac{\nu_{1}+\nu_{2}}{2}+i+1\right)\left\{\int_{1}^{\infty} \frac{u_{2}^{\frac{\nu_{1}}{2}+i-1}\left(u_{2}-1\right)}{\left(u_{2}+\frac{\nu_{2}}{\nu_{1}}\right)^{\frac{\nu_{1}+\nu_{2}}{2}+i+1}} d u_{2}\right.
\end{aligned}
$$




$$
\left.+\int_{\Lambda}^{1} \frac{u_{2}^{\frac{\nu_{1}}{2}+i-1}\left(1-u_{2}\right)}{\left(u_{2}+\frac{\nu_{2}}{\nu_{1}}\right)^{\frac{\nu_{1}+\nu_{2}}{2}+i+1}} d u_{2}\right\}, \quad(\Lambda \leq 1),
$$

and

(5.16) $E\left\{\left(Q_{1}-\frac{\nu_{1}}{\nu_{2}} Q_{3}\right)^{2}, D_{(b)}\right\}=\sum_{i} C_{i}\left(\frac{\nu_{2}}{\nu_{1}}\right)^{\frac{\nu_{2}}{2}}\left(2 \sigma^{2}\right)^{\frac{\nu_{1}+\nu_{2}}{2}+i+2} \Gamma\left(\frac{\nu_{1}+\nu_{2}}{2}+i+2\right)$

$$
\begin{aligned}
& \cdot \int_{\Lambda}^{\infty} \frac{u_{2}^{\frac{\nu_{1}}{2}+i-1}\left(u_{2}+\frac{\nu_{2}}{\nu_{1}}\right)^{\frac{\nu_{1}+2}{2}+i+2}}{\left(u_{2}-1\right)^{2}} d u_{2},(\Lambda \geq 1) \\
& =\sum_{i} C_{i}\left(\frac{\nu_{2}}{\nu_{1}}\right)^{\frac{\nu_{2}}{\nu_{2}}}\left(2 \sigma^{2}\right)^{\frac{\nu_{1}+\nu_{2}}{2}+i+2} \Gamma\left(\frac{\nu_{1}+\nu_{2}}{2}+i+2\right)\left\{\int_{1}^{\infty} \frac{u_{2}^{\frac{\nu_{1}}{2}+i-1}}{\left(u_{2}+\frac{\nu_{2}}{\nu_{1}}\right)^{\left.-\frac{\nu_{2}+\nu_{2}}{2}+1\right)^{2}}} d u_{2}\right. \\
& \left.-\int_{\Lambda}^{1} \frac{u_{2}^{\frac{\nu_{1}}{2}+i-1}\left(1-u_{2}\right)^{2}}{\left(u_{2}+\frac{\nu_{2}}{\nu_{1}}\right)^{\frac{\nu_{1}+\nu_{2}}{2-i+2}}} d u_{2}\right\}, \quad(\Lambda \leq 1)
\end{aligned}
$$

Corollary 5.1. Specially, when $K=0$ and $\Lambda=1$ (this case may be adopted very often in our routine), $E\left\{\hat{\sigma}_{t}^{2}\right\}$ and $E\left\{\left(\hat{\sigma}_{t}^{2}\right)^{2}\right\}$ becomes simple.

Indeed we have

$$
\begin{aligned}
E\left\{\hat{\sigma}_{t}^{2}\right\} & =\frac{2 \sigma^{2}}{\left(\nu_{1}+\nu_{2}\right)} \sum_{i} \frac{e^{-\lambda} \lambda^{i} \Gamma\left(\frac{\nu_{1}+\nu_{2}}{2}+i+1\right)}{i ! \Gamma\left(\frac{\nu_{2}}{2}\right) \Gamma\left(\frac{\nu_{1}}{2}+i\right)}\left[B\left(\frac{\nu_{1}}{2}+i+1, \frac{\nu_{2}}{2}\right)\right. \\
& \left.-B_{1-\phi^{\prime \prime}}\left(\frac{\nu_{1}}{2}+i+1, \frac{\nu_{2}}{2}\right)-\frac{\nu_{1}}{\nu_{2}}\left\{B\left(\frac{\nu_{1}}{2}+i, \frac{\nu_{2}}{2}+1\right)-B_{1-\phi^{\prime \prime}}\left(\frac{\nu_{1}}{2}+i, \frac{\nu_{2}}{2}+1\right)\right\}\right],
\end{aligned}
$$

and

$$
\begin{aligned}
& \text { (5.19) } E\left\{\left(\hat{\sigma}_{l}^{2}\right)^{2}\right\}=\frac{\left(2 \sigma^{2}\right)^{2}}{\left(\nu_{1}+\nu_{2}\right)^{2}} \sum_{i} \frac{e^{-\lambda} \lambda^{i} \Gamma\left(\frac{\nu_{1}+\nu_{2}}{2}+i+2\right)}{i ! \Gamma\left(\frac{\nu_{2}}{2}\right) \Gamma\left(\frac{\nu_{1}}{2}+1\right)}\left[\nu _ { 2 } \left\{B\left(\frac{\nu_{1}}{2}+i+2, \frac{\nu_{2}}{2}\right)\right.\right. \\
& \left.-B_{1-\phi^{\prime \prime}}\left(\frac{\nu_{1}}{2}+i+2, \frac{\nu_{2}}{2}\right)\right\}-2 \nu_{1}\left\{B\left(\frac{\nu_{1}}{2}+i+1, \frac{\nu_{2}}{2}+1\right)-B_{1-\phi^{\prime \prime}}\left(\frac{\nu_{1}}{2}+i+1, \frac{\nu_{2}}{2}+1\right)\right\}
\end{aligned}
$$




$$
+\frac{\nu_{1}^{2}}{\nu_{2}}\left\{B\left(\frac{\nu_{1}}{2}+i, \frac{\nu_{2}}{2}+2\right)-B_{1-\phi^{\prime \prime}}\left(\frac{\nu_{1}}{2}+i, \frac{\nu_{2}}{2}+2\right) !\right] \text {. }
$$

Corollary 5.2. Let us consider in particular an inference procedure which is defined by $A=0$. This corresponds to the case when we use always an estimate $\left(Q_{1}-\frac{\nu_{1}}{\nu_{2}} Q_{3}\right)\left(\nu_{1}+\nu_{2}\right)$ for the non-centricity parameter $2 \sigma^{2} \lambda /$ $\left(\nu_{1}+\nu_{2}\right)$.

We have then the following expectation,

$$
\begin{aligned}
E\left\{\hat{\sigma}_{t}^{2}\right\}= & \frac{2 \sigma^{2}}{\left(\nu_{1}+\nu_{2}\right)}\left[\lambda-\sum_{i} \frac{e^{-\lambda} \lambda^{i} \Gamma\left(\frac{\nu_{1}+\nu_{2}}{2}+i+1\right)}{i ! \Gamma\left(\frac{\nu_{2}}{2}\right) \Gamma\left(\frac{\nu_{1}}{2}+i\right)}\left\{B_{\phi^{\prime \prime}}, \frac{\nu_{1}}{2}+i+1, \frac{\nu_{2}}{2}\right)-\frac{\nu_{1}}{\nu_{2}}\right. \\
& \left.\left.\cdot B_{\phi^{\prime \prime}}\left(\frac{\nu_{1}}{2}+i, \frac{\nu_{2}}{2}+1\right)\right\}\right],
\end{aligned}
$$

and further $E\left\{\left(\hat{\sigma}_{t}^{2}\right)^{2}\right\}$ is given by (5.13) as the special case when $\phi^{\prime}=0$.

From Corollary 5.2, we can observe that our technique of ordinary estimation of the non-centricity parameter after the analysis of variance has certain bias.

Corollary 5.3. When we adopt an inference procedure defined by Corollary 5.2., the probability that we obtain “ minus" $\hat{\sigma}_{i}^{2}$ is given by"

$$
1-\sum_{i} \frac{e^{-\lambda} \lambda^{i}}{i !} I_{1-\downarrow^{\prime \prime}}\left(\frac{\nu_{1}}{2}+i, \frac{\nu_{2}}{2}\right)
$$

\subsection{Type 6: (The inference of variance after a preliminary F-test)}

Let $O_{n_{1}}:\left(x_{11}, x_{12}, \cdots, x_{1 n_{1}}\right)$ be a random sample of $n_{1}$ from a normal population $N\left(\mu_{1}, \sigma_{1}^{2}\right)$, and let $O_{n_{2}}:\left(x_{21}, x_{22}, \cdots, x_{2_{2}}\right)$ be another random sample of $n_{2}$ drawn from another normal population $N\left(\mu_{2}, \sigma_{2}^{2}\right)$. The values of these two variances, $\sigma_{1}^{2}$ and $\sigma_{2}^{2}$, are unknown to us and the distinction between these two values $\sigma_{1}^{2}$ and $\sigma_{2}^{2}$ may be regarded as hypothetical, and we are in the situation of having an incompletely specified model in the sense defined in Type 2 .

Our rule of the statistical procedure is formulated in the following way:

(i) Let $s_{j}^{2}$ be sample unbiassed variance mutually independently defined by $s_{j}^{2}=\sum_{i}\left(x_{j i}-\bar{x}_{j}.\right) / \nu_{j}$ with $\nu_{j}\left(=n_{j}-1\right)$ degrees of freedom, $(j=1,2)$.

(ii) Let the statistic $F_{0}$ be defined by $F_{0}=s_{2}^{2} / s_{1}^{2}$.

(iii) Let us define the statistic $\hat{\sigma}^{2}$ in the following way, (a) $\hat{\sigma}^{2}=s_{2}^{2}$, if $F_{0} \leq \lambda$, 
(b) $\hat{\sigma}^{2}=\left(\nu_{1} s_{1}^{2}+\nu_{2} s_{2}^{2}\right) /\left(\nu_{1}+\nu_{2}\right)$, if $\lambda<F_{0}<\Lambda$,

(c) $\hat{\sigma}^{2}=s_{1}^{2}$, if $F_{0} \geq A$,

where $\lambda$ and $A$ are the non-negative prescribed constants.

There are two important practical problems $\left(1^{\circ}\right)$ and $\left(2^{\circ}\right)$ to which our present statistical procedure can be applied with certain respective different interpretation.

$\left(1^{\circ}\right)$ The first problem is concerned with an estimation of the minimum value of $\sigma_{1}^{2}$ and $\sigma_{2}^{2}$. The current estimate based upon our intuition would be $\min \left(s_{1}^{2}, s_{2}^{2}\right)$. This should not exclude, however, any other estimate which may be more natural and more effective under our situation of incompletely specified model. Indeed the distinction between $\sigma_{1}^{2}$ and $\sigma_{2}^{2}$ is hypothetical in our present situation, and we may and we shall appeal to the pooled estimate of the common value of the population variances in order to aim at the improvemerit of the precision of our estimate, when the hypothesis $H_{0}$ : $\sigma_{1}^{2}=\sigma_{2}^{2}$ can not be rejected.

$\left(2^{\circ}\right)$ The second problem arises under the situation when we have a proof based on physical grounds which can secure us to assume $\sigma_{1}^{2} \leq \sigma_{2}^{2}$. For instance, the first population is concerned with a test-mixture on which observations are more carefully done than those on a control corresponding to the first population, and we can assume $\sigma_{1}^{2} \leq \sigma_{2}^{2}$. The procedure (a) implies the rejection of the statistic $s_{1}^{2}$ which is contradictory to our assumption $\sigma_{1}^{2} \leq \sigma_{2}^{2}$.

Theorem 6.1. The distribution function of $\hat{\sigma}^{2}$ is given by

$$
\begin{gathered}
\operatorname{Pr} .\left\{\hat{\sigma}^{2}<u\right\}=\int_{0}^{u} \int_{0}^{\lambda} g_{2}\left(u_{1}, u_{2}\right) d u_{1} d u_{2}+\frac{1}{\nu_{1}+\nu_{2}}\left\{\nu_{1} \int_{0}^{u} \int_{\lambda}^{\Lambda} g_{1}\left(u_{1}, u_{3}\right) d u_{1} d u_{3}+\nu_{2}\right. \\
\left.\cdot \int_{0}^{u} \int_{\lambda}^{\Lambda} g_{2}\left(u_{1}, u_{2}\right) d u_{1} d u_{2}\right\}+\int_{0}^{u} \int_{\Lambda}^{\infty} g_{1}\left(u_{1}, u_{3}\right) d u_{1} d u_{3},
\end{gathered}
$$

where we put

$$
\begin{aligned}
& g_{1}\left(u_{1}, u_{3}\right)=2 \frac{-\frac{\nu_{1}+\nu_{2}}{2}}{\frac{\nu_{1}}{\sigma^{2}} \frac{\nu_{1}}{2}\left(\frac{\nu_{2}}{\sigma^{2}}\right)^{\frac{\nu_{2}}{2}}} u_{1}^{\left.\frac{\nu_{1}}{2}\right) \Gamma\left(\frac{\nu_{2}}{2}\right)} u_{1}^{\frac{\nu_{2}}{2}-1} u_{3}^{\frac{\nu_{1}+\nu_{2}}{2}-1} \exp \left\{-\frac{u_{3}}{2}\left(\frac{\nu_{1}}{\sigma_{1}^{2}}+\frac{u_{1} \nu_{2}}{\sigma_{2}^{2}}\right)\right\}, \\
& g_{2}\left(u_{1}, u_{2}\right)=2^{-\frac{\nu_{1}+\nu_{2}}{2}} \frac{\left(\frac{\nu_{1}}{\sigma_{1}^{2}}\right)^{\frac{\nu_{1}}{2}}\left(\frac{\nu_{2}}{\sigma_{2}^{2}}\right)^{\frac{\nu_{2}}{2}}}{\Gamma\left(\frac{\nu_{1}}{2}\right) \Gamma\left(\frac{\nu_{2}}{2}\right)} u_{1}^{-\frac{\nu_{1}}{2}-1} u_{2}^{\frac{\nu_{1}+\nu_{2}}{2}-1} \exp \left\{-\frac{u_{2}}{2}\left(\frac{\nu_{1}}{\sigma_{1}^{2} u_{1}}+\frac{\nu_{2}}{\sigma_{2}^{2}}\right)\right\} .
\end{aligned}
$$

Proof : The joint distribution of $s_{1}^{2}$ and $s_{2}^{2}$ is given and we put $u_{1}=$ $s_{2}^{2} / s_{1}^{2}, u_{2}=s_{2}^{2}$ and $u_{3}=s_{1}^{2}$, and then we transform $\left(s_{1}^{2}, s_{2}^{2}\right)$ of the joint distribution to $\left(u_{1}, u_{2}\right)$ and $\left(u_{1}, u_{3}\right)$, where the Jacobian is given by 


$$
\frac{\partial\left(s_{1}^{2}, s_{2}^{2}\right)}{\partial\left(n_{1}, u_{2}\right)}=\frac{u_{2}}{u_{1}^{2}}, \quad \frac{\partial\left(s_{1}^{2}, s_{2}^{2}\right)}{\partial\left(u_{1}, u_{3}\right)}=u_{3}
$$

Now the right side of (6.1) can be obtained easily.

Theorem 6.2. The mean value $E\left\{\hat{\sigma}^{2}\right\}$ and the mean square error M.S.E. $\left\{\hat{\sigma}^{2}\right\}$ are given by

(6.3) $E\left\{\hat{\sigma}_{2}\right\}=\sigma_{1}^{2}\left\{1-I_{\Theta}\left(\frac{\nu_{2}}{2}, \frac{\nu_{1}}{2}+1\right)\right\}+\sigma_{2}^{2} I_{\theta}\left(\frac{\nu_{1}}{2}, \frac{\nu_{2}}{2}+1\right)+\frac{\nu_{1} \sigma_{1}^{2}}{\nu_{1}+\nu_{2}}\left\{I_{\Theta}\left(\frac{\nu_{2}}{2}, \frac{\nu_{1}}{2}+1\right)\right.$

$$
\left.-I_{\theta}\left(\frac{\nu_{2}}{2}, \frac{\nu_{1}}{2}+1\right)\right\}+\frac{\nu_{2} \sigma_{2}^{2}}{\nu_{1}+\nu_{2}}\left\{I_{\Theta}\left(\frac{\nu_{1}}{2}, \frac{\nu_{2}}{2}+1\right)-I_{\theta}\left(\frac{\nu_{1}}{2}, \frac{\nu_{2}}{2}+1\right)\right\},
$$

and

(6.4) M.S.E. $\left\{\hat{\sigma}_{2}\right\}=E\left\{\left(\hat{\sigma}^{2}-\sigma_{1}^{2}\right)^{2}\right\}$

$$
\begin{aligned}
= & \sigma_{1}^{4}\left(1+\frac{2}{\nu_{1}}\right)\left\{1-I_{\Theta}\left(\frac{\nu_{2}}{2}, \frac{\nu_{1}}{2}+2\right)\right\}+\sigma_{2}^{4}\left(1+\frac{2}{\nu_{2}}\right) I_{\theta}\left(\frac{\nu_{1}}{2}, \frac{\nu_{2}}{2}+2\right) \\
& +\frac{\nu_{1}^{2}}{\left(\nu_{1}+\nu_{2}\right) \sigma_{1}^{2}} \sigma_{1}^{4}\left(1+\frac{2}{\nu_{1}}\right)\left\{I_{\Theta}\left(\frac{\nu_{2}}{2}, \frac{\nu_{1}}{2}+2\right)-I_{\theta}\left(\frac{\nu_{2}}{2}, \frac{\nu_{1}}{2}+2\right)\right\}+\frac{\nu_{2}^{2}}{\left(\nu_{1}+\nu_{2}\right)^{2}} \sigma_{2}^{4}\left(1+\frac{2}{\nu_{2}}\right) \\
& \cdot\left\{I_{\Theta}\left(\frac{\nu_{1}}{2}, \frac{\nu_{2}}{2}+2\right)-I_{\theta}\left(\frac{\nu_{1}}{2}, \frac{\nu_{2}}{2}+2\right)\right\}+\frac{2 \nu_{1} \nu_{2}}{\left(\nu_{1}+\nu_{2}\right)^{2}} \sigma_{1}^{2} \sigma_{2}^{2}\left\{I_{\Theta}\left(\frac{\nu_{1}}{2}+1, \frac{\nu_{2}}{2}+1\right)-I_{\theta}\left(\frac{\nu_{1}}{2}+1, \frac{\nu_{2}}{2}+1\right)\right. \\
& -2 \sigma_{1}^{2}\left[\sigma_{2}^{2} I_{\theta}\left(\frac{\nu_{1}}{2}, \frac{\nu_{2}}{2}+1\right)-\sigma_{1}^{2} I_{\Theta}\left(\frac{\nu_{1}}{2}, \frac{\nu_{2}}{2}+1\right)+\frac{\nu_{1} \sigma_{1}^{2}}{\nu_{1}+\nu_{2}}\left\{I_{\Theta}\left(\frac{\nu_{2}}{2}, \frac{\nu_{1}}{2}+1\right)-I_{\theta}\left(\frac{\nu_{2}}{2}, \frac{\nu_{1}}{2}+1\right)\right\}\right. \\
& \left.+\frac{\nu_{2} \sigma_{2}^{2}}{\nu_{1}+\nu_{2}}\left\{I_{\Theta}\left(\frac{\nu_{1}}{2}, \frac{\nu_{2}}{2}+1\right)-I_{\theta}\left(\frac{\nu_{1}}{2}, \frac{\nu_{2}}{2}+1\right)\right\}\right]-\sigma_{1}^{4},
\end{aligned}
$$

where we put

$$
\Theta=\frac{\sigma_{1}^{2} \nu_{2} \Lambda}{\sigma_{2}^{2} \nu_{1}+\sigma_{1}^{2} \nu_{2} \Lambda}, \quad \theta=\frac{\sigma_{1}^{2} \nu_{2} \lambda}{\sigma_{2}^{2} \nu_{1}+\sigma_{1}^{2} \nu_{2} \lambda} .
$$

Proof: The mean value and the mean square error are calculated by

$$
E\left\{\hat{\sigma}_{2}\right\}=E\left\{u_{2} \mid D_{(a)}\right\}+E\left\{\frac{\nu_{1} u_{3}+\nu_{2} u_{2}}{\nu_{1}+\nu_{2}} \mid D_{(b)}\right\}+E\left\{u_{3} D_{(c)}\right\},
$$

and

$$
\begin{aligned}
E\left\{\left(\hat{\sigma}^{2}-\sigma_{1}^{2}\right)^{2}\right\}= & E\left\{\left(u_{2}-\sigma_{1}^{2}\right)^{2} \mid D_{(a)}\right\}+E\left\{\left(\frac{\nu_{1} u_{3}+\nu_{2} u_{2}}{\nu_{1}+\nu_{2}}-\sigma_{1}^{2}\right)^{2} \mid D_{(b)}\right\} \\
& +E\left\{\left(u_{3}-\sigma_{1}^{2}\right)^{2} \mid D_{(c)}\right\},
\end{aligned}
$$

where $D_{(a)}: u_{1} \leq \lambda, D_{(b)}: \lambda<u_{1}<A$ and $D_{(c)}: u_{1} \geq A$. 
With regard to the mean square $E\left\{u_{2}^{2}\right\}, E\left\{u_{3}^{2}\right\}$ and $E\left\{u_{2} u_{3}\right\}$, we can obtain the following results in a general domain $B \leq u_{1} \leq A$,

$$
\begin{aligned}
& E\left\{u_{2}^{2}\right\}=\sigma_{2}^{4}\left(1+\frac{2}{\nu_{2}}\right)\left\{I_{\alpha}\left(\frac{\nu_{2}}{2}, \frac{\nu_{1}}{2}+2\right)-I_{\beta}\left(\frac{\nu_{2}}{2}, \frac{\nu_{1}}{2}+2\right)\right\}, \\
& E\left\{u_{3}^{2}\right\}=\sigma_{1}^{4}\left(1+\frac{2}{\nu_{1}}\right)\left\{I_{\alpha}\left(\frac{\nu_{1}}{2}, \frac{\nu_{2}}{2}+2\right)-I_{\beta}\left(\frac{\nu_{1}}{2}, \frac{\nu_{2}}{2}+2\right)\right\},
\end{aligned}
$$

and

$$
E\left\{u_{2} u_{3}\right\}=\sigma_{1}^{2} \sigma_{2}^{2}\left\{I_{\alpha}\left(\frac{\nu_{1}}{2}+1, \frac{\nu_{2}}{2}+1\right)-I_{\beta}\left(\frac{\nu_{1}}{2}+1, \frac{\nu_{2}}{2}+1\right)\right\},
$$

where we put

$$
\alpha=\frac{\nu_{2} \sigma_{1}^{2} A}{\nu_{1} \sigma_{2}^{2}+\nu_{2} \sigma_{1}^{2} A}, \quad \beta=\frac{\nu_{2} \sigma_{1}^{2} B}{\nu_{1} \sigma_{2}^{2}+\nu_{2} \sigma_{1}^{2} B}
$$

Then, in our problem, we may displace $\alpha$ and $\beta$ for $\Theta$ and $\theta$, that is,

$$
\begin{array}{ll}
D_{(a)}: & \alpha=\theta, \beta=0 \\
D_{(b)}: & \alpha=\Theta, \beta=\theta \\
D_{(c)}: & \alpha=1, \beta=\Theta .
\end{array}
$$

Consequently (6.3) and (6.4) are obtained.

Corollary 6.1. Specially, an inference procedure defined by the " never pooling", that is, $\lambda=0$ and $A=0$ shows us that $E\left\{\hat{\sigma}^{2}\right\}=\sigma_{1}^{2} . M . S . E .\left\{\hat{\sigma}^{2}\right\}=$ $\frac{2}{\nu} \sigma_{1}^{4}$. An inference procedure defined by the "always pooling", that is, $\lambda=0$ and $A=\infty$, shows us that $E\left\{\hat{\sigma}_{2}\right\}=\left(\nu_{1} \sigma_{1}^{2}+\nu_{2} \sigma_{2}^{2}\right) /\left(\nu_{1}+\nu_{2}\right)$,

and

$$
\text { M.S.E. }\left\{\hat{\sigma}^{2}\right\}=\left(\frac{\nu_{1} \sigma_{1}^{2}+\nu_{2} \sigma_{2}^{2}}{\nu_{1}+\nu_{2}}\right)^{2}+\frac{2\left(\nu_{1} \sigma_{1}^{4}+\nu_{2} \sigma_{2}^{4}\right)}{\left(\nu_{1}+\nu_{2}\right)^{2}}-2 \sigma_{1}^{2} \frac{\nu_{1} \sigma_{1}^{2}+\nu_{2} \sigma_{2}^{2}}{\nu_{1}+\nu_{2}}+\sigma_{1}^{4} .
$$

Corollary 6.2. Specially when $\lambda=0, E\left\{\hat{\sigma}^{2}\right\}$ and M.S.E. $\left\{\hat{\sigma}^{2}\right\}$ become

$$
E\left\{\hat{\sigma}^{2}\right\}=\sigma_{1}^{2}\left\{1-\frac{\nu_{2}}{\nu_{1}+\nu_{2}} I_{\Theta}\left(\frac{\nu_{2}}{2}, \frac{\nu_{1}}{2}+1\right)\right\}+\frac{\nu_{2}}{\nu_{1}+\nu_{2}} \sigma_{2}^{2} I_{\Theta}\left(\frac{\nu_{1}}{2}, \frac{\nu_{2}}{2}+1\right)
$$

and

(6.15) M.S.E. $\left\{\hat{\sigma}^{2}\right\}=\frac{1}{\left(\nu_{1}+\nu_{2}\right)^{2}}\left\{\nu_{1}\left(\nu_{1}+2\right) I_{\Theta}\left(\frac{\nu_{2}}{2}, \frac{\nu_{1}}{2}+2\right) \sigma_{1}^{4}+\nu_{2}\left(\nu_{2}+2\right) I_{\Theta}\left(\frac{\nu_{1}}{2}, \frac{\nu_{2}}{2}+2\right) \sigma_{2}^{4}\right.$

$$
\left.+2 \nu_{1} \nu_{2} \sigma_{1}^{2} \sigma_{2}^{2} I_{\Theta}\left(\frac{\nu_{1}}{2}+1, \frac{\nu_{2}}{2}+1\right)\right\}+\frac{2 \nu_{2}}{\nu_{1}+\nu_{2}}\left\{\sigma_{1}^{4} I_{\Theta}\left(\frac{\nu_{2}}{2}, \frac{\nu_{1}}{2}+1\right)-\sigma_{2}^{4} I_{\Theta}\left(\frac{\nu_{1}}{2}, \frac{\nu_{2}}{2}+1\right)\right\}
$$




$$
+\sigma_{1}^{4}\left(1+\frac{2}{\nu_{1}}\right)\left\{1-I_{\Theta}\left(\frac{\nu_{2}}{2}, \frac{\nu_{1}}{2}+2\right)\right\}-\sigma_{1}^{4}
$$

These results coincide with the Bancroft's ones [1].

\section{$\S$ 4. Further discussion and conclusion}

The application of statistical procedures in which we have both a validity check of observations and a consideration of our previous informations before making any use of current statistical inferences are very often and even customarily used in our routine of statistical analysis. These reflect our practical situations where our models are not completely specified. The present paper is concerned with successive processes of statistical inferences to be used in these situations which may happen in biological and pharmaceutical sciences as well as in physical scierices.

The paper discusses with six fundamental types of these statistical procedures. Our main work is to formulate accurately our statistical procedures and then to evaluate the mean value and the mean square error of each of our statistics defined by these statistical procedures. The numerical aspects are quite important for any practical applications of our statistical procedures. The author of the present paper is now preparing the use of our magnetic drum data-processing in Shionogi Pharmaceutical Company for the calculations of numerical tables associated to statistical procedures discussed in this paper.

The remaining problems are concerned with the choices of two switching constants $\lambda$ and $A$. We are not certain at this moment whether or not the values of $\lambda$ and $A$ can be duly determined merely from the view-point of minimizing the mean square error, which depend upon the values of $\lambda$ and A. Furthermore we are not certain whether or not any application of minimax strategy may be successful approach in determining the choices of $\lambda$ and $A$. The essential difficulties arise in the situations where our models are incompletely specified in view of our previous informations. There are many different situations for which incompletely specified models can be introduced with different implications. The choice of $\lambda$ and $A$ are deeply connected with the design of screening experiments in the sense of G.E.P. Box. It is hoped that our formulations of several fundamental statistical procedures and allied calculations of the moments of our statistics will serve for further studies.

Kyushu University and Shionogi Pharmaceutical Co. 


\section{References}

Bahadur, R. R. and RobBins, H. [1] : The problem of the greater mean, Ann. Math. Stat., 21 (1950), 469-487.

BancRoft, T. A. [1] : On biases in estimation due to the use of preliminary tests of significance, Ann. Math. Stat., 15 (1944), 190-204.

BenNet, B. D. [1] : Estimation of means on the basis of preliminary tests of significance, Ann. Inst. Stat. Math., Tokyo, 4 (1952), 31-43.

Bozivioh, H., Bancroft, T. A. and Hartley, H. O. [1] : Power of analysis of variance test procedures for certain incompletely specified models, I, Ann. Math. Stat., 27 (1956), 1017-1043.

Kitagawa, T. [1] : Successive process of statistical inferences, Mem. Facu. of Sci. Kyushu Univ., Ser. A, 5 (1950), 139-180.

Kitagawa T. [2] : Successive process of statistical inferences (5), Mem. Facu. of Sci. Kyushu Univ., Ser. A, 7 (1953), 81-106. 